\title{
16. GEOCHEMISTRY OF INTERSTITIAL WATERS AND SEDIMENTS, LEG 64, GULF OF CALIFORNIA ${ }^{1}$
}

Joris M. Gieskes, ${ }^{2}$ Henry Elderfield, ${ }^{3}$ James R. Lawrence, ${ }^{4}$ Jeff Johnson, ${ }^{2}$ Barbara Meyers, ${ }^{2}$ and Andrew Campbell ${ }^{2}$

\begin{abstract}
Studies of interstitial waters obtained from DSDP Leg 64 drill sites in the Gulf of California have revealed information both on early diagenetic processes in the sediments resulting from the breakdown of organic matter and on hydrothermal interactions between sediments and hot doleritic sill intrusions into the sediments.

In all the sites drilled sulfate reduction occurred as a result of rapid sediment accumulation rates and of relatively high organic carbon contents; in most sites methane production occurred after sulfate depletion. Associated with this methane production are high values of alkalinity and high concentrations of dissolved ammonia, which causes ion exchange processes with the solid phases leading to intermediate maxima in $\mathrm{Mg}^{++}, \mathrm{K}^{+}, \mathrm{Rb}^{+}$, and $\mathrm{Sr}^{++}($?). Though this phenomenon is common in Leg 64 drill sites, these concentration reversals had been noticed previously only in Site 262 (Timor Trough) and Site 440 (Japan Trench).

Penetrating, hot dolerite sills have led to substantial hydrothermal alteration in sediments at sites drilled in the Guaymas Basin. Site 477 is an active hydrothermal system in which the pore-water chemistry typically shows depletions in sulfate and magnesium and large increases in lithium, potassium, rubidium, calcium, strontium, and chloride. Strontium isotope data also indicate large contributions of volcanic matter and basalt to the pore-water strontium concentrations. At Sites 478 and 481 dolerite sill intrusions have cooled to ambient temperatures but interstitial water concentrations of $\mathrm{Li}^{+}, \mathrm{Rb}^{+}, \mathrm{Sr}^{++}$, and $\mathrm{Cl}^{-}$show the gradual decay of a hydrothermal signal that must have been similar to the interstitial water chemistry at Site 477 at the time of sill intrusion. Studies of oxygen isotopes of the interstitial waters at Site 481 indicate positive values of $\delta^{18} \mathrm{O}$ (SMOW) as a result of high-temperature alteration reactions occurring in the sills and the surrounding sediments.

A minimum in dissolved chloride at about 100-125 meters sub-bottom at Sites 478,481 , and particularly Site 479 records a possible paleosalinity signal, associated with an event that substantially lowered salinities in the inner parts of the Gulf of California during Quaternary time.
\end{abstract}

\section{INTRODUCTION}

Drilling by Glomar Challenger in the Gulf of California (Fig. 1) presented a unique opportunity to study in detail the geochemical processes affecting sediments and interstitial waters in an area of rapid sediment accumulation, relatively high heat flow, and ocean floor genesis. Because of the nearness of the Gulf to terrigenous sediment sources as well as the high productivity in the surface waters, sedimentation rates are high throughout (van Andel and Shor, 1964). In sediments of this nature, diagenetic processes involving the bacterial decomposition of organic carbon generally leave characteristic imprints on the chemical composition of the interstitial waters (Berner, 1980; Gieskes, 1975, 1981). Elevated heat flow, characteristic of extensional basins, can be expected to affect the diagenetic processes occurring in the sediments, in particular the diagenesis of biogenic silica to more stable silica phases (Kastner et al., 1977). Finally, drilling on or near the observed high heat flow anomalies found in the Guaymas Basin (Lawver et al., 1975) would provide an opportunity to test theories of hydrothermal circulation associated with basaltic magma generation in an extensional basin characterized by very high

\footnotetext{
${ }^{1}$ Curray, J. R., Moore, D. G., et al., Init. Repts. DSDP, 64: Washington (U.S. Govt. Printing Office).

2 Scripps Institution of Oceanography, La Jolla, California.

3 Department of Earth Sciences, The University, Leeds, England LS2 9JT.

${ }^{4}$ Lamont-Doherty Geological Observatory, Palisades, New York.
}

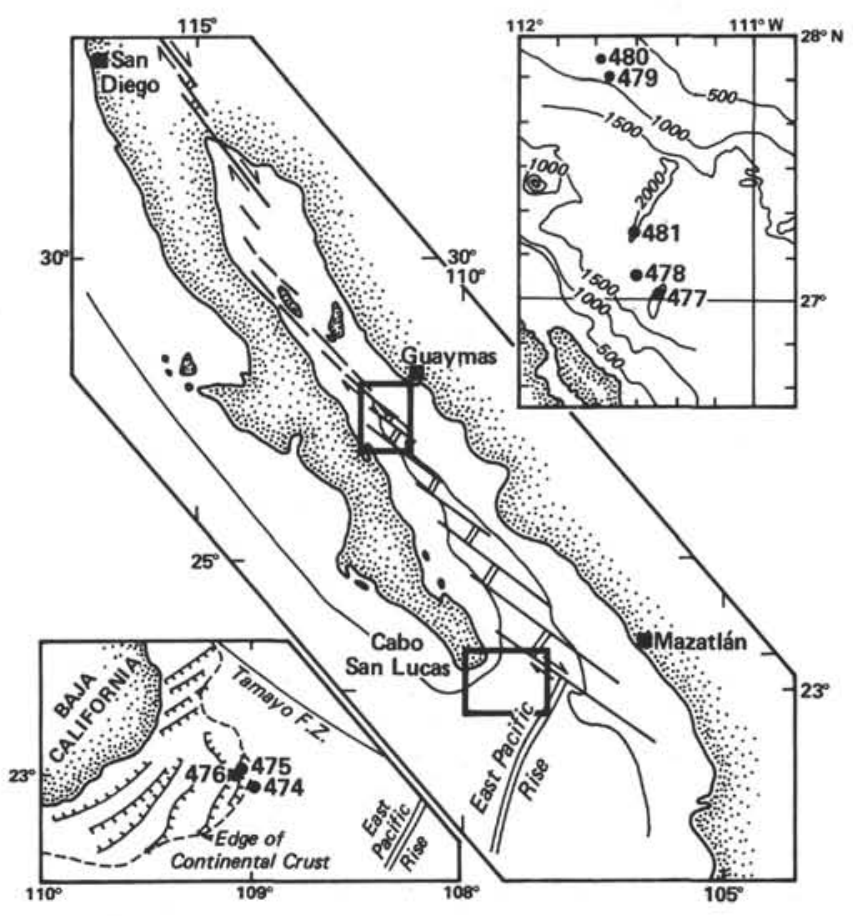

Figure 1. The Gulf of California and Leg 64 site locations.

rates of sediment accumulation (Moore, 1973; Williams et al., 1979; Einsele et al., 1980).

In this paper we present the results of the study of the chemical composition of the interstitial waters and 
of sediments obtained during Leg 64 . Interpretations are necessarily preliminary in nature, but the data will be used to delineate some general, observable trends that will help in understanding the geochemical processes affecting sediments in the Gulf of California.

\section{METHODS}

\section{Interstitial Waters}

In these studies interstitial waters were obtained by routine shipboard squeezing of sediment samples (Manheim and Sayles, 1974), usually effected within a very short time after retrieval of the cores. No special attention was given to temperature-of-squeezing effects (Sayles et al., 1973; Gieskes, 1973, 1974; Sayles and Manheim, 1975), though such effects may be of importance in sediments such as those obtained at Site 477 , where temperatures at the bottom of the hole may exceed $200^{\circ} \mathrm{C}$ in situ. Samples obtained at this site, particularly in the deeper sections, have suffered to greater or lesser extents from seawater contamination, which would again have complicated attempts to simulate in situ conditions $\left(200^{\circ} \mathrm{C}, 200-250\right.$ atmospheres of pressure). In order to correct for this contamination we have assumed that magnesium would be absent from the pore waters below 150 meters sub-bottom depth.

In order to check on the validity of the routine interstitial water program, in situ samples (Barnes et al., 1979) were taken at Site 477 (one sample), Site 479 (three samples), and Site 481 (one sample). These data will be compared with shipboard samples in the discussion of observations at each drill site.

The shipboard program included $p \mathrm{H}$, alkalinity, salinity, chloride, calcium, magnesium, silica, phosphate, and ammonia (J.M.G.). All subsequent analyses were carried out in our shore laboratories.

Methods are documented in Table 1. For rubidium a new method was developed; it is described in the Appendix to this chapter.

\section{Bulk Sediments}

For the chemical analysis of the bulk sediments we utilized wet chemical methods similar to those described by Donnelly and Wallace (1976). Sediment standards were those prepared by E. R. Sholkovitz, which were then standardized against U.S.G.S. standard rocks. In addition we analyzed U.S.G.S. Standard AGV-1 as an unknown. Generally, quantities of $20 \mathrm{mg}$ or less of ground-up material were used in these analyses. Though weighing errors, particularly when smaller quantities of material are involved, may not be trivial, atomic ratios of the elements with aluminum as the referent will be little affected. A comparison of the atomic ratios for AGV-1 (Table 5) indicates that with the exception of $\mathrm{Mg} / \mathrm{Al}$ a good agreement is generally obtained with the reported values of Flanagan (1972).

For the determination of manganese and lithium and the solid ${ }^{87} \mathrm{Sr} /{ }^{86} \mathrm{Sr}$ ratio, we digested $0.5 \mathrm{~g}$ samples in hydrogen fluoride in Teflon bombs using a modification of the method of Shapiro and Brannock (1962). Determinations were carried out by atomic absorption

Table 1. Methods used in interstitial water analyses.

\begin{tabular}{lll}
\hline Component & \multicolumn{1}{c}{ Method } & \multicolumn{1}{c}{ Reference } \\
\hline $\begin{array}{l}\text { pH } \\
\text { Alkalinity }\end{array}$ & Titration & Gieskes, 1973, 1974 \\
Salinity & Refractometer & Gieskes, 1974 \\
Chanheim and Sayles, 1974 \\
Chloride & Titration & Gieskes, 1974 \\
Calcium & Titration & Gieskes and Lawrence, 1976 \\
Magnesium & Titration & Gieskes and Lawrence, 1976 \\
Silica & Colorimetric & Mann and Gieskes, 1975 \\
Phosphate & Colorimetric & Gieskes, 1973, 1974 \\
Ammonia & Colorimetric & Gieskes, 1973, 1974 \\
Lithium & Atomic absorption & Gieskes and Johnson, 1981a \\
Rubidium & Flameless A.A. & Appendix \\
Potassium & Atomic absorption & Gieskes, 1974 \\
Strontium & Atomic absorption & Gieskes, 1974 \\
Manganese & Atomic absorption & Gieskes, 1974 \\
$87 \mathrm{Sr} / 86 \mathrm{Sr}$ & Mass spectrometry & Hawkesworth and Elderfield, 1978 \\
$18 \mathrm{O} / 16 \mathrm{O}$ & Mass spectrometry & Epstein and Mayeda, 1953 \\
\hline
\end{tabular}

techniques using the method of internal addition for the Li data. ${ }^{87} \mathrm{Sr} /{ }^{86} \mathrm{Sr}$ measurements were made by mass spectrometry.

Interstitial water data are presented in Tables 2, 3, and 10 (Site 477 data corrected for sea-water contamination); data on the solids are presented in Tables 4-8.

As yet, no salt corrections have been made to the bulk sediment data, but these corrections will be small $(<5 \%)$ even for constituents such as $\mathrm{K}$ and $\mathrm{Mg}$. Further work on this is in progress, but elemental atomic ratios presented in this paper will be little affected.

\section{DISCUSSION}

In our discussion, we will first address the observations made at each site and will follow with a more general discussion of particular problems.

Since sedimentation rates are of great importance in all these discussions, a generalized sedimentation rate diagram is presented in Figure 2.

\section{Site 474 (Figs. 3 and 4)}

Quaternary sedimentation rates at this site have been very high ( $>260 \mathrm{~m} / \mathrm{m} . \mathrm{y}$.) and thus it is not surprising that sulfate reduction is completed below 10 meters depth into the sediments. Below this depth methane production is common (Simoneit, this volume, Pt. 2). Associated with these processes of bacterial degeneration of organic matter are large increases in alkalinity, ammonia, and dissolved phosphate. As a result of the large increase in dissolved ammonia, significant ion exchange occurs with the clay minerals of the sediments, leading to maxima in dissolved magnesium at about 90 meters. Slight increases in dissolved rubidium and potassium and in strontium are also related to these ion exchange processes. Similar maxima have been observed previously at Site 262 in the Timor Trough (Cook, 1974) and at Site 440 in the Japan Trench (Moore and Gieskes, 1980).

Dissolved silica values show a gradual increase with depth in the upper 270 meters of sediments. Below this point a sharp decrease in dissolved silica coincides with a lithologic change in the sediments that results from the reconstitution of biogenic opal-A to opal-CT in the form of silicified claystones. This break in lithology is comparable to that associated with the silicification front discussed by Kastner and Gieskes (1976) for Site 323 in the Bellingshausen Abyssal Plain. Important to note here are the dissolved calcium, magnesium, and potassium profiles, which are again similar to those observed at Site 323. Kastner and Gieskes (1976) showed that in the latter site the silicification reactions were associated with the alteration of plagioclase (source of $\mathrm{Ca}$ ) and the formation of smectites (sink for $\mathrm{Mg}$ ) and of $\mathrm{K}$-feldspar (sink for $\mathrm{K}$ ). We suggest that similar reactions also occur at Site 474. A possible volcanic source for $\mathrm{Ca}$ is indicated by the parallel decrease in the ${ }^{87} \mathrm{Sr} /$ ${ }^{86} \mathrm{Sr}$ ratio in dissolved strontium to values well below those of contemporaneous sea water (Hawkesworth and Elderfield, 1978). In addition, a minimum occurs in $\delta^{18} \mathrm{O}$ (Fig. 16), indicating involvement of volcanic matter alteration (Gieskes and Lawrence, in press).

Dissolved lithium appears unaffected by the ion exchange processes that have just been noted for other cations. A rather major source of lithium is located below 
Table 2. Interstitial water data, Leg 64.

\begin{tabular}{|c|c|c|c|c|c|c|c|c|c|c|c|c|c|c|c|c|c|}
\hline $\begin{array}{c}\text { Sample } \\
\text { (interval in } \mathrm{cm} \text { ) }\end{array}$ & $\mathbf{z}$ & $p \mathrm{H}$ & $\begin{array}{c}\text { Alk } \\
\text { (meq/1) }\end{array}$ & $\begin{array}{c}\mathrm{SO}_{4} \\
(\mathrm{mM})\end{array}$ & $\begin{array}{l}\mathrm{NH}_{4} \\
(\mathrm{mM})\end{array}$ & $\begin{array}{l}\mathrm{PO}_{4} \\
(\mu \mathrm{M})\end{array}$ & $\begin{array}{l}\mathrm{SiO}_{2} \\
(\mu \mathrm{M})\end{array}$ & $\underset{(\mathrm{mM})}{\mathrm{Ca}}$ & $\begin{array}{c}\mathrm{Mg} \\
(\mathrm{mM})\end{array}$ & $\underset{(\mu \mathrm{M})}{\mathrm{Sr}}$ & $\underset{(\mu \mathrm{M})}{\mathrm{Mn}}$ & $\underset{(\mu \mathrm{M})}{\mathrm{Li}}$ & $\underset{(\mathrm{mM})}{\mathrm{K}}$ & $\begin{array}{c}\mathrm{Rb} \\
(\mu \mathrm{M})\end{array}$ & ${ }^{87} \mathrm{Sr} /{ }^{86} \mathrm{Sr}$ & $\begin{array}{l}\mathrm{Cl} \\
(\% 0)\end{array}$ & $\underset{(\%)}{S}$ \\
\hline Hole 474 & & & & & & & & & & & & & & & & & \\
\hline $1-1,90-94$ & 1 & 7.65 & 3.93 & 28.1 & & & & & & 87 & 29.2 & 27 & 11.4 & & & & \\
\hline $2-1,140-150$ & 4 & 7.38 & 17.4 & 16.0 & 0.94 & 41 & 830 & 7.45 & 48.7 & 96 & 0.6 & 29 & 11.0 & 2.7 & & 19.2 & 34.6 \\
\hline $3-2,140-150$ & 13 & 7.40 & 34.9 & 0.0 & 3.55 & 119 & 925 & 4.67 & 45.1 & 127 & 0 & 46 & 10.4 & 2.4 & & 19.2 & 34.1 \\
\hline $5-2,140-150$ & 33 & 7.31 & 33.6 & 0.2 & 4.15 & 104 & 885 & 4.86 & 44.1 & 125 & 0 & 45 & 10.5 & 2.0 & & 19.1 & 34.1 \\
\hline $7-3,0-10$ & 44 & 7,21 & 37.5 & 0.2 & 6.50 & (25) & 1065 & 4.63 & 40.6 & 148 & 0 & 45 & 10.9 & 2.4 & & 19.2 & 34.1 \\
\hline $11-2,81-87$ & 90 & & 71.5 & 0 & 12.80 & 115 & 1020 & 3.26 & 51.3 & 196 & 0 & & 15.0 & 3.4 & 0.70916 & 19.2 & 36.3 \\
\hline $13-1,41-47$ & 108 & & & 0 & 13.9 & 102 & & 2.98 & 46.7 & & & & & & & 19.2 & 36.3 \\
\hline $16-2,95-106$ & 138 & 7.71 & 59.6 & 0 & 13.8 & 81 & 1095 & 4.75 & 39.2 & 205 & 0 & 104 & 14.8 & 2.8 & & 18.9 & 35.2 \\
\hline $17-5,140-150$ & 150 & & 52.5 & 0 & (18.5) & 44 & 1030 & 5.10 & 38.2 & 203 & 0 & 123 & 14.6 & 2.4 & 0.70910 & 19.0 & 34.9 \\
\hline Hole 474A & & & & & & & & & & & & & & & & & \\
\hline $1-2,140-150$ & 165 & 7.23 & 54.2 & & 15.0 & & 1190 & 5.80 & 35.7 & 199 & 8 & 144 & 13.4 & 2.5 & & 19.0 & 35.2 \\
\hline $4-5,140-150$ & 197 & 7.22 & 50.0 & & 14.5 & 40 & 1105 & 7.22 & 35.1 & 194 & 12 & 186 & 12.6 & 2.2 & & 19.3 & 34.6 \\
\hline $7-2,140-150$ & 224 & 7.02 & 43.9 & & 14.1 & 28.5 & 1270 & 9.49 & 33.2 & 185 & 7.4 & 268 & 11.0 & 1.3 & 0.70879 & 19.3 & 34.4 \\
\hline $10-1,140-150$ & 250 & & 35.9 & 0 & 12.7 & 12 & 1300 & 12.2 & 30.5 & 190 & 11.2 & 329 & 9.7 & 1.5 & & 19.2 & 34.1 \\
\hline $13-3,140-150$ & 280 & 6.99 & 23.4 & & 10.6 & 4.5 & 780 & 16.2 & 26.8 & 191 & 13.3 & 464 & 5.8 & 1.4 & & 19.5 & 33.8 \\
\hline $16-4,140-150$ & 310 & 7.17 & 16.3 & & 7.8 & 12.5 & 480 & 19.1 & 22.8 & 207 & 15.5 & 527 & 2.7 & 0.95 & 0.70840 & 19.4 & 33.6 \\
\hline $19-1,140-150$ & 330 & 7.06 & 12.95 & & 6.5 & 11.5 & 520 & 19.65 & 25.0 & 193 & 25.0 & 551 & 2.4 & 0.95 & 0.70823 & 19.4 & 33,6 \\
\hline $23-3,140-150$ & 368 & 7.14 & 10.8 & 0 & 5.7 & 4.5 & 540 & 24.9 & 19.5 & 219 & 30.8 & 606 & 1.4 & 1.4 & 0.70796 & 19.4 & 33.3 \\
\hline $27-4,140-150$ & 405 & 7.33 & 4.65 & & 4.25 & 16 & 320 & 24.8 & 14.1 & 246 & 5.4 & 614 & 1.7 & 1.25 & 0.70789 & 19.4 & 33.0 \\
\hline $30-2,140-150$ & 431 & 7.32 & 4.85 & & 3.60 & 21 & 370 & 27.8 & 12.6 & 309 & 8.6 & 666 & 1.3 & 1.4 & 0.70792 & 19.3 & 33.0 \\
\hline $33-3,140-150$ & 462 & 7.33 & 5.20 & 0 & 3.05 & 4.5 & 360 & 27.2 & 13.6 & 281 & 14.0 & 456 & 1.9 & 1.25 & 0.70788 & 19.3 & 33.0 \\
\hline $36-1,140-150$ & 490 & & & & 2.9 & 8 & 195 & 28.8 & 12.2 & & & 417 & & 1.3 & & 19.0 & 32.7 \\
\hline $39-1,140-150$ & 516.5 & & & & 2.7 & 12 & 95 & 32.5 & 9.5 & & & 428 & & & 0.70765 & 19.1 & 31.9 \\
\hline $40-4,20-30$ & 530 & & & & 1.9 & 1.5 & 95 & 44.8 & 4.1 & & & 273 & & 1.3 & & 18.9 & 32.2 \\
\hline $41-4,140-150$ & 539 & & & 0 & & 7 & 130 & 40.9 & 8.5 & & & 270 & & 1.4 & 0.70793 & 19.4 & 33.3 \\
\hline Hole 475 & & & & & & & & & & & & & & & & & \\
\hline $1-1,140-150$ & 2 & & & & 0.07 & 4 & 628 & 10.06 & 52.7 & & & & & & & $(18.9)$ & 34.9 \\
\hline $2-4,140-150$ & 12 & 7.58 & 6.12 & 26.8 & 0.36 & 21 & 700 & 10.12 & 50.7 & 86 & 30.2 & 27 & 11.2 & 1.8 & & 19.1 & 34.9 \\
\hline $3-4,140-150$ & 21 & 7.48 & 11.8 & & 0.79 & 32 & 865 & 9.08 & 48.9 & 100 & 25.9 & & 10.5 & & & 19.2 & 34.9 \\
\hline $5-3,140-150$ & 39 & 7.38 & 15.5 & 18.5 & 1.14 & 31 & 850 & 8.75 & 49.2 & 81 & 20.2 & & 10.1 & & & 19.3 & 35.2 \\
\hline $7-1,140-150$ & 55 & 7.40 & 16.7 & 15.5 & 1.31 & 24 & 840 & 9.12 & 47.6 & 95 & 20.2 & & 9.3 & & & 19.2 & 34.9 \\
\hline $9-3,140-150$ & 75 & 7.24 & 18.3 & 12.5 & 1.41 & 19 & 1035 & 10.00 & 46.5 & 96 & 33.6 & 77 & 9.7 & 1.3 & & 19.3 & 34.9 \\
\hline $11-5,140-150$ & 99 & 7.30 & 16.6 & 13.1 & 1.41 & 9 & 970 & 11.24 & 44.8 & 104 & 23.2 & & 8.7 & & & 19.3 & 34.9 \\
\hline $13-3,140-150$ & 116 & 7.14 & 14.9 & 15.3 & 1.32 & 6 & 1090 & 12.71 & 45.1 & 108 & 20.0 & 118 & 7.8 & 1.3 & & 19.3 & 34.9 \\
\hline $15-4,140-150$ & 135 & 7.01 & 11.2 & 19.5 & 1.25 & 7 & 1170 & 14.90 & 43.3 & III & 13.1 & 128 & 8.0 & 1.3 & & 19.3 & 34.9 \\
\hline $17-1,140-150$ & 153 & 6.98 & 9.2 & 20.3 & 1.08 & 4.5 & 1440 & 16.04 & 43.7 & 109 & 9.4 & & 7.2 & & & 19.2 & 35.2 \\
\hline Hole 475A & & & & & & & & & & & & & & & & & \\
\hline $1-5,140-150$ & 6 & 7.47 & 5.3 & 28.7 & & 21 & & 10.35 & 51.1 & 86 & 24.3 & 27 & 9.6 & 1.8 & & 19.1 & \\
\hline Hole 476 & & & & & & & & & & & & & & & & & \\
\hline $1-3,140-150$ & 6 & 7.46 & 15.6 & 18.3 & 1.11 & 59 & 835 & 7.55 & 50.6 & 87 & 9.4 & & 10.4 & & & 19.06 & 35.2 \\
\hline $2-2,140-150$ & 12 & 7.35 & 22.2 & & 1.58 & 98 & 840 & 5.96 & 50.2 & 100 & 6.2 & 46 & 11.1 & & 0.70915 & 19.25 & 34.9 \\
\hline $3-5,140-150$ & 27.5 & 7.45 & 26.7 & 3.9 & 2.06 & 75 & 950 & 4.83 & 46.7 & 88 & 0 & 43 & I1.4 & & & 19.25 & 34.1 \\
\hline $5-3,140-150$ & 42.5 & 7.39 & 26.8 & 1.6 & 2.40 & 68 & 900 & 4.77 & 44.9 & 88 & 0 & 40 & 10.3 & & & 19.19 & 34.0 \\
\hline $7-6,140-150$ & 61 & 7.20 & 22.5 & 1.6 & 2.58 & 35 & 985 & 5.70 & 40.4 & 93 & 6.3 & 121 & 9.5 & & 0.70886 & 19.28 & 33.8 \\
\hline $9-4,140-150$ & 80 & 7.29 & 21.8 & 3.2 & 2.39 & 25 & 980 & 6.89 & 42.2 & 93 & 2.5 & & 9.8 & & & 19.31 & 33.8 \\
\hline $11-4,140-150$ & 100 & 7.19 & 19.4 & 7.5 & 2.06 & 16 & 1055 & 10.28 & 42.8 & 97 & 9.7 & 193 & 9.1 & & & 19.22 & 34.4 \\
\hline $12-4,140-150$ & 110 & 7.28 & 18.1 & 9.1 & 1.86 & iI & 1120 & 11.72 & 43.6 & 104 & 12.6 & & 8.3 & & 0.70872 & 19.16 & 34.4 \\
\hline $13-5,140-150$ & 120 & 7.19 & 16.2 & 11.6 & 1.73 & 5.5 & 1075 & 15.42 & 43.8 & 109 & 15.4 & & 8.7 & & & 19.22 & 34.4 \\
\hline $15-2,140-150$ & 135 & 7.17 & 16.2 & 16.6 & 1.28 & 2 & 965 & 18.18 & 45.0 & 122 & 18.4 & 226 & 8.1 & & & 19.15 & 35.2 \\
\hline $17-2,140-150$ & 152 & 7.17 & 9.9 & 22.4 & 1.04 & 2 & 1275 & 19.11 & 44.9 & 119 & 24.1 & & 7.2 & & & 19.25 & 35.5 \\
\hline $18-4,140-150$ & 167 & 6.94 & 9.0 & 22.6 & 0.91 & 2 & 1320 & 20.79 & 44.8 & 109 & 24.0 & & 6.9 & & & 19.19 & 36.0 \\
\hline $20-5,140-150$ & 186 & 7.21 & 6.97 & 24.4 & 0.67 & 1 & 1110 & 20.8 & 46.4 & 107 & 17.4 & & 6.1 & & 0.70866 & 19.25 & 35.8 \\
\hline $21-4,0-10$ & 193 & 7.34 & 4.64 & 27.7 & 0.49 & 0 & 360 & 22.7 & 46.4 & 113 & 22.9 & & 5.3 & & & 19.22 & 36.0 \\
\hline $26-1,18-22$ & 238 & & & & & & & 23.8 & 46.7 & & & & & & & 19.04 & 35.8 \\
\hline Hole 477 & & & & & & & & & & & & & & & & & \\
\hline $2-3,140-150$ & 6 & 7.58 & 38.3 & 14.5 & 2.03 & 185 & 970 & 5.88 & 48.5 & 96 & 42.3 & 15.6 & 10.2 & 1.9 & & 19.05 & 34.1 \\
\hline $3-1,140-150$ & 12 & 7.57 & 46.6 & 10.0 & 3.60 & 158 & 980 & 4.28 & 49.5 & 102 & 35.9 & 13 & 10.0 & 2.5 & 0.70891 & 19.05 & 34.3 \\
\hline $4-1,133-140$ & 21.5 & 7.33 & 60.1 & 9.3 & 8.40 & 186 & 1320 & 5.63 & 54.7 & 90 & 17.1 & 18 & 10.6 & 1.35 & & 19.23 & 36.3 \\
\hline $5-1,140-150$ & 32.5 & 7.28 & 56.5 & 11.3 & 10.90 & 188 & 1300 & 5.11 & 56.4 & 108 & 38.2 & 23 & 10.3 & 2.25 & & 19.17 & 36.2 \\
\hline In situ & 48 & & 6.2 & 14.6 & 1.57 & 0 & 570 & 9.93 & 46.3 & 112 & 18.9 & 159 & 13.1 & 3.5 & & 19.42 & 35.2 \\
\hline $7-1,142-150$ & 51.5 & & 11.3 & 21.9 & 2.80 & 0 & 1310 & 10.07 & 39.6 & 167 & 13.5 & (147) & 8.4 & & 0.70859 & 19.74 & 34.1 \\
\hline $7-2,27-32$ & 52 & & & & 2.03 & 20 & 1420 & 9.60 & 38.7 & & & & & & & & 34.1 \\
\hline $15-1,126-131$ & 106 & 6.97 & 6.8 & 23.7 & 0.93 & 0 & 740 & 16.29 & 51.2 & 121 & 72.8 & 192 & 7.4 & 3.5 & & 20.24 & 37.1 \\
\hline $16-4,140-150$ & 120 & 6.00 & 6.3 & 17.0 & & 0 & 1780 & 22.6 & 23.4 & 154 & 313 & 273 & 19.1 & 12 & 0.70771 & 20.74 & 36.6 \\
\hline $17-2,140-150$ & 126 & 6.05 & 8.9 & 4.6 & & 0 & 1815 & 30.8 & 2.8 & 186 & 295 & 413 & 20.8 & 16 & 0.70705 & 21.15 & 35.5 \\
\hline $19-1,140-150$ & 146 & 6.48 & 9.6 & 0.6 & & 0 & 2500 & 50.2 & 0.7 & 312 & 92 & 693 & 30.6 & 38 & 0.70635 & 21.18 & 36.8 \\
\hline $20-1,140-150$ & 155 & 6.23 & 13.8 & 4.1 & & 0 & 1905 & 39.0 & 17.7 & 204 & 122 & 424 & 26.0 & 27.3 & 0.70658 & 20.58 & 36.6 \\
\hline $20-2,71-76$ & 156 & & & & & 0 & 1680 & 44.5 & 1.4 & & & & & & & 21.24 & \\
\hline $22-1,140-150$ & 173 & 6.74 & 11.1 & 8.5 & & 0 & 2050 & 26.7 & 30.7 & 143 & 119 & & 22.7 & 25.3 & 0.70655 & 20.18 & 36.6 \\
\hline $23, \mathrm{CC}$ & 185 & & & & & 0 & 1200 & 30.9 & 26.3 & & & & & 27.3 & & 20.74 & 36.6 \\
\hline Hole 477A & & & & & & & & & & & & & & & & & \\
\hline $5-1,114-120$ & 195 & & & & 11.3 & & & 36.4 & 2.2 & & & 851 & 89 & & & 20.96 & \\
\hline $5-1,114-120$ & 195 & & & & 11.0 & & & 35.5 & 4.95 & & & & & 56.5 & & 21.24 & \\
\hline $6, \mathrm{CC}$ & 210 & & & & 4.3 & & & 25.0 & 25.4 & 152 & & & 23.5 & 20.5 & 0.7064 ? & 20.21 & \\
\hline $7-1,3-7$ & 212 & & & & 10.8 & & & 35.9 & 4.7 & & & & & & & 21.12 & \\
\hline $8, \mathrm{CC}$ & 229 & & & & 8.9 & & & 38.3 & 6.3 & & & & & & & - & \\
\hline $9-1,69-71$ & 231 & & & & 0.3 & & & 12.5 & 45.14 & & & & & 3.7 & & 17.53 & \\
\hline $10-1,110-120$ & 240 & & & & 10.2 & & & 35.1 & 8.34 & 193 & & & 36.5 & 47 & 0.70560 & 20.93 & \\
\hline $10-1,110-120$ & 240 & & & & 6.6 & & & 28.2 & 21.09 & & & 495 & 34.0 & 33 & & 20.21 & \\
\hline
\end{tabular}


Table 2. (Continued).

\begin{tabular}{|c|c|c|c|c|c|c|c|c|c|c|c|c|c|c|c|c|}
\hline $\begin{array}{c}\text { Sample } \\
\text { (interval in } \mathrm{cm} \text { ) }\end{array}$ & z & $p \mathrm{H}$ & $\underset{\text { (meq/l) }}{\text { Alk }}$ & $\begin{array}{l}\mathrm{SO}_{4} \\
(\mathrm{mM})\end{array}$ & $\begin{array}{l}\mathrm{NH}_{4} \\
(\mathrm{mM})\end{array}$ & $\begin{array}{l}\mathrm{PO}_{4} \\
(\mu \mathrm{M})\end{array}$ & $\begin{array}{l}\mathrm{SiO}_{2} \\
(\mu \mathrm{M})\end{array}$ & $\underset{(\mathrm{mM})}{\mathrm{Ca}}$ & $\begin{array}{c}\mathrm{Mg} \\
(\mathrm{mM})\end{array}$ & $\underset{(\mu \mathrm{M})}{\mathrm{Sr}}$ & $\begin{array}{c}\mathrm{Mn} \\
(\mu \mathrm{M})\end{array}$ & $\underset{(\mu \mathrm{M})}{\mathrm{Li}}$ & $\underset{(\mathrm{mM})}{\mathrm{K}}$ & $\begin{array}{c}\mathrm{Rb} \\
(\mu \mathrm{M})\end{array}$ & ${ }^{87} \mathrm{Sr} /{ }^{86} \mathrm{Sr}$ & $\underset{(\%)}{\mathrm{Cl}}$ \\
\hline Hole 478 & & & & & & & & & & & & & & & & \\
\hline $1-3,135-150$ & 3 & 8.15 & 11.15 & 25.3 & 0.9 & 48 & 830 & 10.07 & 52.4 & 83 & 166.5 & & 11.4 & & & 19.12 \\
\hline $2-4,140-150$ & 10 & 7.83 & 13.54 & 26.6 & 1.8 & 72 & 740 & 9.00 & 50.9 & 86 & 27 & 19.2 & 12.1 & & & 19.19 \\
\hline $3-4,140-150$ & 19.5 & 7.58 & 17.33 & 7.9 & 2.2 & 84 & 840 & 8.27 & 51.5 & 81 & 15.1 & & 11.5 & & & 19.09 \\
\hline $4-5,140-150$ & 31 & 7.63 & 20.7 & 17.8 & 2.45 & 81 & 860 & 8.14 & 52.4 & 84 & 12.5 & 23 & 9.5 & & 0.70875 & 19.09 \\
\hline $5-4,140-150$ & 39.5 & 7.63 & 21.5 & 17.4 & 2.7 & 84 & 910 & 7.95 & 51.3 & 92 & 6.2 & & 10.2 & & & 19.16 \\
\hline $6-4,140-150$ & 48 & 7.64 & 20.7 & 16.6 & 2.7 & 78 & 960 & 7.89 & 51.8 & 92 & 9.9 & & 10.2 & & & 18.96 \\
\hline $7-5,140-150$ & 60 & 7.66 & 23.9 & 13.0 & 3.35 & 81 & 870 & 6.85 & 51.8 & 94 & 6.2 & 20 & 9.1 & & & 19.00 \\
\hline $8-4,140-150$ & 68 & 7.54 & 31.0 & 8.3 & 4.4 & 128 & 1010 & 5.32 & $\begin{array}{l}51.9 \\
51.9\end{array}$ & 73 & 10.2 & 20 & 8.5 & & & 18.96 \\
\hline $9-3,140-150$ & 76 & 7.48 & 43.8 & 1.4 & 6.1 & 228 & 1050 & 4.12 & 51.9 & 64 & 10.6 & & 9.6 & & 0.70873 & 19.09 \\
\hline $11-4,110-120$ & 97 & 7.62 & 51.7 & 0 & 8.9 & 216 & 1015 & 3.98 & 50.7 & 96 & 16.2 & 30 & 10.0 & & 0.10072 & 18.96 \\
\hline $13-3,140-150$ & 114 & 7.36 & 56.6 & 1 & 9.7 & 204 & 1190 & 3.56 & 53.4 & 118 & 30.2 & 39 & 11.4 & & & 19.09 \\
\hline $15-2,140-150$ & 131.5 & 7.39 & 44.9 & 1 & 9.5 & 195 & 1220 & 3.41 & 58.3 & 110 & 15.5 & 52 & 9.6 & & & 19.16 \\
\hline $17-3,140-150$ & 150 & 7.38 & 61.5 & i & 8.9 & 219 & 1165 & 3.89 & 57.4 & 90 & 8.2 & 71 & 7.9 & & 0.70877 & 19.28 \\
\hline $19-5,140-150$ & 164 & 7.25 & 60.6 & i & 9.8 & 198 & 1370 & 4.37 & 55.1 & 101 & 8.2 & 89 & 7.2 & & & 19.32 \\
\hline $21-2,140-150$ & 178 & & 50.1 & i & 10.8 & 115 & 1225 & 3.81 & 48.0 & 95 & 12.3 & 120 & 6.8 & & & 19.30 \\
\hline $22-2,140-150$ & 187 & 7.61 & 42.0 & & 10.5 & 63 & 1355 & 5.65 & 41.4 & 99 & 11.7 & 118 & 6.8 & & & (18.50) \\
\hline $28-3,140-150$ & 248 & 7.50 & 9.6 & & 5.9 & 4.5 & 1650 & 16.12 & 23.7 & 142 & 40.7 & 151 & 6.8 & & & 19.22 \\
\hline $29-2,140-150$ & 253 & 7.84 & 7.8 & & 5.3 & 0 & 1350 & 17.27 & 18.7 & 132 & 21.3 & 118 & 5.8 & & 0.70828 & 19.28 \\
\hline $30-2,0-10$ & 259.5 & & 8.4 & & 5.1 & 0 & 1475 & 17.19 & 21.4 & 146 & 21.3 & $\begin{array}{l}234 \\
234\end{array}$ & 7.1 & & 0.10020 & 19.34 \\
\hline $33-1,140-150$ & 280 & 7.92 & 10.8 & & 3.35 & 0 & 1560 & 19.22 & 21.8 & 162 & 27.4 & 202 & 5.9 & & & 19.19 \\
\hline $36-2,140-150$ & 310 & & 9.3 & & 3.3 & 0 & 1525 & 20.83 & 21.2 & 190 & 27.1 & 145 & 5.9 & & & 19.41 \\
\hline $39-2,140-150$ & 328 & & . & & $>3.5$ & 0 & 780 & (18.67) & (23.5) & & & 120 & .3 & & & (19.62) \\
\hline $40-1,140-150$ & 338 & 7.45 & 6.2 & & 6.1 & 0 & 475 & 17.23 & 19.3 & 150 & 26.4 & 126 & 7.5 & & 0.70822 & 19.31 \\
\hline Hole 479 & & & & & & & & & & & & & & & & \\
\hline $1-1,140-150$ & 2 & 7.56 & 46.0 & & 2.8 & 186 & 970 & 6.07 & 50.4 & & & & & & & 19.12 \\
\hline $3-1,135-145$ & 14 & 7.65 & 81.1 & 3.5 & 10.0 & 288 & 920 & 5.05 & 53.8 & 115 & 14.8 & 76 & 10.4 & & & 18.97 \\
\hline $5-3,110-120$ & 34 & & 76.5 & 4.5 & 9.6 & 275 & 1180 & 5.59 & 54.9 & 112 & 14.5 & 76 & 10.1 & & & 19.09 \\
\hline $7-5,140-150$ & 55 & 7.01 & 75.3 & 4.0 & 14.5 & 111 & 890 & 5.23 & 39.6 & 142 & 14.2 & 404 & 11.2 & & 0.70920 & 18.68 \\
\hline $8-3,140-150$ & 63 & & 71.3 & & 15.6 & 86 & 1160 & 4.37 & 34.3 & 181 & 16.5 & 525 & 13.9 & & & 18.72 \\
\hline $9-2,140-150$ & 72 & 6.96 & 70.1 & 3.3 & 17.2 & 47 & 1200 & 5.44 & 33.9 & 159 & 8.7 & & 11.4 & & & 18.81 \\
\hline $11-2,140-150$ & 90 & 6.92 & 72.3 & & 17.7 & 38 & 1250 & 7.77 & 34.3 & & & 602 & & & & 18.78 \\
\hline In situ 1 & 99 & & & & 13.1 & 0 & 1030 & 8.08 & 35.2 & & & 500 & & & & 18.65 \\
\hline $13-1,140-150$ & 110 & 6.85 & 66.7 & 3.2 & 20.9 & 30 & 1270 & 8.39 & 28.0 & 180 & 3.4 & 705 & 11.6 & & & 18.72 \\
\hline $15-5,140-150$ & 130 & 6.86 & 62.0 & & 23.3 & 28 & 1390 & 9.57 & 23.7 & 155 & 13.2 & & 11.7 & & 0.70907 & \\
\hline $17-5,140-150$ & 149 & 6.80 & 56.4 & & 23.4 & 51 & 1390 & 10.11 & 20.0 & 209 & 12.7 & & 12.4 & & & 18.81 \\
\hline In situ II & 165 & & & & 19.4 & 53 & 1120 & 10.80 & & & & 718 & & & & \\
\hline $19-4,140-150$ & 170 & 6.91 & 52.3 & & 24.6 & 56 & 1400 & 10.19 & 19.0 & 202 & 7.8 & 900 & 12.1 & & & 18.62 \\
\hline $21-4,140-150$ & 188 & 6.95 & 48.4 & 1.4 & 26.0 & 24 & 1520 & 10. & 17.4 & 214 & & 1040 & 11.5 & & & 18.81 \\
\hline $24-4,140-150$ & 212 & 6.84 & 44.7 & 2.8 & 26.2 & 45 & 1620 & 11.07 & 19.1 & 221 & 0 & 990 & 12.5 & & & 18.84 \\
\hline In situ III & 231.5 & & & & 7.6 & 66 & & & 17.5 & & & 800 & & & & \\
\hline $27-4,140-150$ & 245 & 6.83 & 38.1 & & 25.6 & 21 & 1555 & 10.88 & 16.7 & 219 & 0 & 990 & 11.8 & & & 19.12 \\
\hline $32-4,140-150$ & 283 & & 33.7 & 3.4 & 24.4 & 20 & 1700 & 11.94 & 16.7 & 204 & 0 & 1000 & 10.9 & & 0.70912 & 19.06 \\
\hline $36-4,140-150$ & 330 & 6.79 & 30.2 & & 22.4 & 24 & 1560 & 12.02 & 14.5 & 223 & 0 & 1080 & 9.1 & & & 19.03 \\
\hline $39-4,140-150$ & 360 & 6.79 & 27.7 & 2.8 & 23.6 & 24 & 1930 & 11.28 & 12.7 & 226 & 0 & 1075 & 8.6 & & 0.70905 & 19.18 \\
\hline $43-1,140-150$ & 395 & & & & & 15 & 1375 & 15.30 & 12.5 & & & 1226 & & & & 19.03 \\
\hline $44-4,140-150$ & 409 & & 23.9 & & 18.6 & 13 & 970 & 15.85 & 14.9 & 285 & 0 & 1040 & 7.7 & & 0.70910 & 19.03 \\
\hline $47-4,140-150$ & 438 & & 17.3 & & 14.5 & 20 & 820 & 14.81 & 15.5 & 275 & 0 & & 5.7 & & 0.70894 & 19.12 \\
\hline Hole 481 (HPC) & & & & & & & & & & & & & & & & \\
\hline $1-1,131-135$ & 1 & & 1 & & 1.0 & 113 & 825 & 9.69 & 50.3 & 116 & 0 & & .2 & .3 & & 97 \\
\hline & 6.3 & 7.74 & & 15.3 & 2.5 & 98 & 875 & 5.99 & 52,1 & 82 & 2.9 & 42 & 10.2 & 3 & & 18.97 \\
\hline $3-1,145-150$ & 11.0 & 7.46 & 76.2 & 7.3 & 6.4 & 222 & & 4.15 & 59.6 & 100 & 0 & & 10.1 & 1.3 & 0.70905 & 19.06 \\
\hline & 15.8 & (7.51) & 92.6 & 3.7 & 9.0 & 236 & 950 & 3. & 65 & 108 & 0 & & 12 & 2.1 & & 19.03 \\
\hline & 25.0 & & 74.9 & & 11.2 & 280 & 855 & 3. & 63.7 & 121 & 0 & & 10. & 2.1 & & 19.06 \\
\hline $144-150$ & 31.5 & 7.77 & 56 & 3.6 & 8.3 & 212 & 935 & 1. & 51.8 & 100 & 0 & 29 & 10. & 2.6 & 0.70905 & 18.97 \\
\hline $8-2,145-150$ & 36 & & 47.9 & & 8.3 & 135 & $\begin{array}{r}7300 \\
1300\end{array}$ & 2. & 49 & 1 & 0 & 29 & 10. & 2.3 & 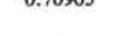 & 18.94 \\
\hline 9 & 39.5 & 7.57 & 48.7 & 3.0 & 5.7 & & 120 & 4 & 48 & 10 & 0 & & is & 1. & & 18 \\
\hline $10-2,1$ & 45.5 & 7.64 & 47.8 & 3.0 & 6.4 & & & & 47 & 8 & 0 & & & 2. & & 15 \\
\hline $11-2,145-150$ & 50.0 & 7.71 & 50.4 & & 6.0 & 116 & 1000 & 2.5 & 47. & 84 & 0 & 22 & 10. & 2. & & 18.97 \\
\hline In situ & 42 & & 49.3 & & 6.5 & 168 & 840 & 2.49 & 50.5 & 111 & 0 & & 10.5 & 1.35 & & 18.97 \\
\hline Hole 481A & & & & & & & & & & & & & & & & \\
\hline $1-1,140$ & 43.5 & 7.61 & 49.5 & & 6.4 & 19 & 84 & 2. & 48. & 94 & 0 & & 12. & 2. & & 18.87 \\
\hline & 64 & 70 & 45 & 2.8 & 9.5 & 26 & 110 & 2 & 51 & 92 & 0 & & 9. & 1. & & 18.94 \\
\hline $4-2,1$ & 74 & 7.40 & 26.5 & (15.9) & 8.5 & 18 & 83 & 4. & 52 & 97 & 0 & 45 & 9. & 1.25 & & 19.00 \\
\hline $5.5,14$ & 86 & 7.45 & 56.4 & 3.6 & 16.1 & 27 & 140 & & & 0 & 0 & 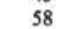 & 11 & & & 18 \\
\hline $6-4,14$ & 94 & 7.44 & 53 & & 15.3 & 263 & 9 & 3. & 47 & 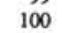 & 0 & 59 & 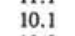 & 23 & 0.70878 & 18.87 \\
\hline $8-5,14$ & 116 & & 45.7 & & & 60 & 110 & & 44 & & 0 & 96 & 10 & & & 19.00 \\
\hline & $\begin{array}{l}110 \\
134\end{array}$ & & 36. & & & ${ }^{\infty} 7.5$ & 100 & & 43 & & 0 & $\begin{array}{l}90 \\
370\end{array}$ & & & 0.70858 & 19.03 \\
\hline & 1. & 7.50 & 29 & & 11 & 0 & 80 & & & & 0 & 1240 & (s & & & 19.09 \\
\hline & is & & 13.6 & & 7 & 0 & 180 & & 32 & 332 & 0 & 870 & 7.0 & 8.5 & 0.70816 & \\
\hline & & & & & 7 & 0 & & & 31 & & & & & & & 19.25 \\
\hline & 20 & & & & 10 & 0 & 600 & 11. & 29 & & 0 & 12 & & & & 20.19 \\
\hline & 22 & & 33.9 & & 17 & 0 & 168 & 8. & 27 & 232 & 0 & 1817 & 5.2 & 4.8 & & 20.13 \\
\hline $2-150$ & 248 & & 36.7 & & 17 & 0 & 15 & 8. & 25. & 201 & 0 & 1466 & 7.4 & 5.0 & & 19.53 \\
\hline $23, \mathrm{CC}$ & 260 & & & & 15 & 0 & 13 & 8. & 25. & & 0 & 1349 & & & & 19.22 \\
\hline $24-5,140-150$ & 268 & & 29.2 & & 14 & 0 & 12 & 13. & 26 & 177 & 0 & 1400 & 8.0 & 3.3 & 0.70830 & 19.59 \\
\hline $25-6,140-150$ & 278 & & 31 . & & is & 0 & 13 & 12 & 27 & & 0 & 546 & 7. & 0.55 & & 19.66 \\
\hline & 28 & & 36. & & & 0 & 12 & & 2. & & 0 & & 8 & 0.65 & 0.70849 & 19.50 \\
\hline & 29 & & & & & 0 & & & & 1 & 0 & 1038 & 8. & $i$. & & \\
\hline $40-150$ & 30 & & 41 & & & 0 & 14 & 14 & 27 & 206 & 0 & & 8. & 1. & & 19.47 \\
\hline $30-5,140-150$ & 325 & & 14.4 & & 10.5 & 0 & 1320 & 15.92 & 22.8 & 171 & 0 & & 8.0 & 1.3 & & 19.59 \\
\hline
\end{tabular}

Note: Blank spaces indicate that no data were obtained. Data in parentheses are considered to be uncertain. 
Table 3. Interstitial water data, Site 477 (corrected for sea-water contamination).

\begin{tabular}{|c|c|c|c|c|c|c|c|c|c|c|c|c|c|c|c|c|}
\hline $\begin{array}{c}\text { Sample } \\
\text { (interval in } \mathrm{cm} \text { ) }\end{array}$ & $\begin{array}{l}\text { Depth } \\
\text { (m) }\end{array}$ & $p \mathrm{H}$ & $\begin{array}{c}\text { Alk } \\
\text { (meq/1) }\end{array}$ & $\begin{array}{l}\mathrm{SO}_{4} \\
(\mathrm{mM})\end{array}$ & $\begin{array}{l}\mathrm{NH}_{4} \\
(\mathrm{mM})\end{array}$ & $\begin{array}{l}\mathrm{PO}_{4} \\
(\mu \mathrm{M})\end{array}$ & $\begin{array}{l}\mathrm{SiO}_{2} \\
(\mu \mathrm{M})\end{array}$ & $\underset{(\mathrm{mM})}{\mathrm{Ca}}$ & $\underset{(\mathrm{mM})}{\mathrm{Mg}}$ & $\underset{(\mu \mathrm{M})}{\mathrm{Sr}}$ & $\begin{array}{c}\mathrm{Mn} \\
(\mu \mathrm{M})\end{array}$ & $\underset{(\mu \mathrm{M})}{\mathrm{Li}}$ & $\underset{(\mathrm{mM})}{\mathrm{K}}$ & $\begin{array}{c}\mathrm{Rb} \\
(\mu \mathrm{M})\end{array}$ & ${ }^{87} \mathrm{Sr} /{ }^{86} \mathrm{Sr}$ & $\begin{array}{c}\mathrm{Cl} \\
(\%)\end{array}$ \\
\hline \multicolumn{17}{|l|}{ Hole 477} \\
\hline $2-3,140-150$ & 6 & 7.58 & 38.3 & 14.5 & 2.0 & 185 & 970 & 5.9 & 48.5 & 96 & 42 & 16 & 10.2 & 1.9 & & 19.05 \\
\hline $3-1,140-150$ & 12 & 7.57 & 46.6 & 10.0 & 3.6 & 158 & 980 & 4.3 & 49.5 & 102 & 36 & 13 & 10.0 & 2.5 & 0.70891 & 19.05 \\
\hline $4-1,133-140$ & 21.5 & 7.33 & 60.1 & 9.3 & 8.4 & 186 & 1320 & 5.6 & 54.7 & 90 & 17 & 18 & 10.6 & 1.4 & & 19.23 \\
\hline $5-1,140-150$ & 32.5 & 7.28 & 56.5 & 11.3 & 10.9 & 186 & 1300 & 5.1 & 56.4 & 108 & 38 & 23 & 10.3 & 2.2 & & 19.17 \\
\hline In situ & 48 & & 6.20 & 14.6 & 1.6 & 0 & & 9.9 & 46.3 & 112 & 19 & 159 & 13.1 & 3.5 & & 19.42 \\
\hline $7-1,142-150$ & 51.5 & & 11.25 & 21.9 & 2.8 & 0 & 1310 & 10.1 & 39.6 & 167 & 14 & 147 & 8.4 & & 0.70859 & 19.74 \\
\hline $7-2,27-32$ & 52 & & & & 2.0 & 20 & 1420 & 9.6 & 38.7 & & & & & & & \\
\hline $15-1,126-131$ & 106 & 6.97 & 6.84 & 23.7 & 0.9 & 0 & 740 & 16.3 & 51.2 & 121 & 73 & 192 & 7.4 & 3.5 & 0.70771 & 20.24 \\
\hline $16-4,140-150$ & 120 & 6.00 & 6.27 & 17.0 & 2.4 & 0 & 1780 & 22.6 & 23.4 & 154 & 313 & 273 & 19.1 & 12 & & 20.74 \\
\hline $17-2,140-150$ & 126 & 6.05 & 8.87 & 4.6 & 4.1 & 0 & 1815 & 30.8 & 2.8 & 186 & 294 & 413 & 20.8 & 16 & 0.70705 & 21.15 \\
\hline $19-1,140-150$ & 146 & 6.48 & 9.58 & 0.6 & 9.2 & 0 & 2500 & 50.2 & 0.7 & 312 & 92 & 693 & 30.6 & 38 & 0.70635 & 21.18 \\
\hline $20-1,140-150$ & 155 & & & $0 ?$ & 10.5 & & 2860 & 53.3 & $0.0^{\mathrm{a}}$ & 261 & 182 & 625 & 33.6 & 41 & 0.7061 & 21.32 \\
\hline $20-2,71-76$ & 156 & & & & 12.2 & & 4872 & 45.4 & $0.0^{\mathrm{a}}$ & & & & & & & 21.30 \\
\hline $22-1,140-150$ & 173 & & & $0 ?$ & 11.2 & & 1680 & 49.1 & $0.0^{\mathrm{a}}$ & 213 & 277 & 927 & 38.9 & 57 & 0.7049 & 21.67 \\
\hline $23, \mathrm{CC}$ & 185 & & & & 11.3 & & 2380 & 50.9 & $0.0^{\mathrm{a}}$ & & & & & 53 & & 22.3 \\
\hline \multicolumn{17}{|l|}{ Hole 477A } \\
\hline $5-1,114-120$ & 195 & & & & 11.9 & & & 37.8 & $0^{\mathrm{a}}$ & & & 851 & 92 & 61 & & 21.25 \\
\hline $6, \mathrm{CC}$ & 210 & & & & 8.3 & & & 38.5 & $0^{\mathrm{a}}$ & 207 & & & 35.1 & 39 & 0.7052 & 21.23 \\
\hline $7-1,3-7$ & 212 & & & & 11.9 & & & 38.4 & $0^{\mathrm{a}}$ & & & & & & & 21.32 \\
\hline $8, \mathrm{CC}$ & 229 & & & & 10.1 & & & 42.1 & $0^{\mathrm{a}}$ & & & & & & & \\
\hline $10-1,110-120$ & 240 & & & & 11.5 & & & 39.9 & $0^{\mathrm{a}}$ & 212 & & 886 & 45 & 54 & 0.7053 & 21.11 \\
\hline
\end{tabular}

Note: Blanks indicate that no data were obtained.

a $\mathrm{Mg}$ assumed to be zero.

270 meters in the zone of silicification. Lithium concentrations in the solid phases (Fig. 4) suggest higher values below 300 meters. If a substantial portion of this lithium is associated with biogenic silica, the reconstitution of opal-A may cause this lithium to be released to the interstitial waters. At lithium concentrations of $45-68 \mathrm{ppm}$ in the solids, less than $5 \%$ release would be able to account for the dissolved lithium increases. Below 400 meters, dissolved lithium shows a large drop that may be associated with uptake reactions in clay minerals during alteration of basalts.

Dolerite sills occur in this site, often showing wide, vertical, calcite veins, especially in the upper sills. We decided to carry out oxygen and carbon isotopic studies on these carbonates (M. Kastner, pers. comm.); the results are presented in Table 9. The data imply "normal" values for the calcites recovered in Sections 474A-42-5 and 474A-44-5-that is, they were formed at relatively low temperatures and with carbonate ions with oceanic $\delta^{13} \mathrm{C}$ values. However, the carbonate veins recovered in Sections 474A-40-2 and 474A-41-5 indicate formation at slightly higher temperatures, with carbon dioxide derived from the decomposition of organic matter. This can be understood in terms of alteration reactions occurring after burial underneath a sediment in which sulfate reduction has led to the production of isotopically light $\left(\delta^{13} \mathrm{C}\right)$ carbon dioxide. Temperatures of formation are lower than presently prevailing temperatures, indicating, presumably, that the calcite veins formed after the cooling of the sill intrusions and the subsequent fracturing. Pore waters containing biogenically produced bicarbonate must then have penetrated these veins and caused the alteration of the basalts, which provided calcium for carbonate precipitation. Typically the interstitial waters below the sills are enriched in dissolved cal- cium and depleted in magnesium as a result of continuing basalt alteration processes.

The chemical composition of the sediments (Fig. 4) shows little systematic changes with depth, so that reactions involving the uptake of magnesium and potassium in these sediments do not appear to be of major importance to that composition. Of course, at sedimentation rates of $260 \mathrm{~m} / \mathrm{m}$.y. the sediment becomes isolated from the overlying ocean below 40-50 meters sub-bottom (Gieskes, 1975), so that a significant influx of potassium and magnesium from the ocean cannot be expected.

\section{Site 475 (Fig. 5)}

At this site sedimentation rates have averaged $\sim 40$ $\mathrm{m} / \mathrm{m}$.y. (Fig. 2). Because of these high accumulation rates and the relatively high organic carbon contents (1-2\%, Simoneit, this volume, Pt. 2), sulfate reduction occurs at this site. A minimum in sulfate is observed at about 100 meters and appears to be the result of increased sulfate reduction in the upper sediment column. Similar minima in dissolved sulfate have been observed at several other sites, for instance, Sites 241, 242 (Gieskes, 1974), and 336 (Gieskes et al., 1979). The decreases in dissolved sulfate are accompanied by the usually observed increases in alkalinity, ammonia, and phosphate, and by a minimum in calcium. Decreases in dissolved magnesium are only minor, and dissolved potassium appears to have a sink in the deeper sections of the site. Dissolved lithium clearly has a source in the sediments, probably in the siliceous materials of the site. Dissolved silica shows a gradual increase with depth, which is probably a result of increased solubilities of opaline silica as a result of the higher temperatures with depth (heat flow $=4 \mathrm{HFU}$ ). 
Table 4. Chemical composition bulk solids, in wt. $\%$, Sites 474,477 , and 479 .

\begin{tabular}{|c|c|c|c|c|c|c|c|c|}
\hline $\begin{array}{c}\text { Sample } \\
\text { (interval in } \mathrm{cm} \text { ) }\end{array}$ & $\begin{array}{l}\text { Depth } \\
\text { (m) }\end{array}$ & $\mathrm{SiO}_{2}$ & $\mathrm{TiO}_{2}$ & $\mathrm{Al}_{2} \mathrm{O}_{3}$ & $\mathrm{Fe}_{2} \mathrm{O}_{3}$ & $\mathrm{MgO}$ & $\mathrm{CaO}$ & $\mathrm{K}_{2} \mathrm{O}$ \\
\hline \multicolumn{9}{|l|}{ Hole 474} \\
\hline $3-2,140-150$ & 13 & 44.4 & 0.43 & 11.12 & 3.92 & 1.59 & 12.83 & 2.53 \\
\hline $16-2,95-106$ & 138 & 45.2 & 0.53 & 12.32 & 4.86 & 2.23 & 11.26 & 2.60 \\
\hline \multicolumn{9}{|l|}{ Hole 474A } \\
\hline $4-5,140-150$ & 197 & 54.1 & 0.63 & 15.09 & 5.30 & 2.34 & 2.21 & 3.26 \\
\hline $16-4,140-150$ & 310 & 53.1 & 0.62 & 14.46 & 6.03 & 2.32 & 4.67 & 4.37 \\
\hline $23-3,140-150$ & 368 & 55.5 & 0.62 & 15.34 & 4.73 & 2.03 & 2.02 & 3.45 \\
\hline $30-2,140-150$ & 431 & 57.2 & 0.58 & 14.03 & 4.87 & 2.02 & 4.77 & 3.20 \\
\hline $39-1,140-150$ & 517 & 52.2 & 0.57 & 14.22 & 4.84 & 2.83 & 1.48 & 3.88 \\
\hline \multicolumn{9}{|l|}{ Hole 477} \\
\hline $2-3,140-150$ & 6 & 55.7 & 0.76 & 13.15 & 4.98 & 2.71 & 3.54 & 2.80 \\
\hline $3-1,140-150$ & 12 & 56.4 & 0.78 & 12.91 & 4.56 & 2.35 & 4.35 & 3.10 \\
\hline $4-1,133-140$ & 21.5 & 53.0 & 0.45 & 9.25 & 3.31 & 2.31 & 5.51 & 1.10 \\
\hline $5-1,140-150$ & 32.5 & 58.0 & 0.61 & 11.03 & 4.13 & 2.24 & 4.42 & 1.90 \\
\hline $7-1,142-150$ & 51.5 & 53.1 & 0.55 & 11.16 & 4.52 & 3.94 & 5.91 & 1.40 \\
\hline $7-2,27-32$ & 52 & 48.3 & 0.75 & 12.95 & 4.83 & 6.14 & 3.81 & 1.10 \\
\hline 9-1, Piece 6 & 60 & 58.4 & 2.03 & 14.55 & 11.39 & 7.25 & 12.51 & 0.10 \\
\hline $15-1,126-131$ & 106 & 34.8 & 0.73 & 14.60 & 5.61 & 11.11 & 0.48 & 3.40 \\
\hline $16-4,140-150$ & 120 & 36.9 & 0.49 & 9.76 & 6.28 & & 13.54 & 1.61 \\
\hline $17-2,140-150$ & 126 & 64.2 & 0.79 & 14.65 & 5.26 & 3.48 & 0.52 & 4.30 \\
\hline $19-1,140-150$ & 146 & 60.3 & 0.80 & 13.11 & 4.25 & 1.64 & 3.96 & 2.95 \\
\hline $20-1,140-150$ & 155 & 76.4 & 0.92 & 19.93 & 6.75 & 3.13 & 3.47 & 4.61 \\
\hline $22-1,140-150$ & 173 & 58.0 & 0.68 & 14.07 & 5.56 & 2.81 & 3.48 & 2.22 \\
\hline $23, \mathrm{CC}$ & 185 & 44.2 & 0.56 & 10.97 & 4.69 & 2.01 & 2.17 & 2.39 \\
\hline \multicolumn{9}{|l|}{ Hole 477A } \\
\hline $5-1,114-120$ & 195 & 59.6 & 0.72 & 13.57 & 4.86 & 2.82 & 4.11 & 0.39 \\
\hline $6, \mathrm{CC}$ & 210 & 41.0 & 0.91 & 17.56 & 17.67 & & 3.46 & 0.40 \\
\hline $7-1,3-7$ & 212 & 64.3 & 0.69 & 13.70 & 3.69 & 1.29 & 3.14 & 0.34 \\
\hline $8, \mathrm{CC}$ & 229 & 62.1 & 0.55 & 12.53 & 6.00 & 3.23 & 5.18 & 0.28 \\
\hline $9-1,69-71$ & 231 & 63.0 & 0.74 & 14.39 & 3.69 & 1.98 & 4.35 & 0.28 \\
\hline $10-1,110-120$ & 240 & 62.2 & 0.67 & 13.15 & 4.62 & 2.58 & 4.77 & 0.28 \\
\hline \multicolumn{9}{|l|}{ Hole 479} \\
\hline $1-1,140-150$ & 2 & 59.0 & 0.43 & 6.44 & 2.86 & 2.36 & 1.27 & 1.55 \\
\hline $3-1,135-145$ & 14 & 54.0 & 0.51 & 10.73 & 4.12 & 2.10 & 3.02 & 2.23 \\
\hline $5-3,110-120$ & 34 & 53.6 & 0.50 & 8.16 & 3.56 & 2.00 & 3.37 & 1.92 \\
\hline $7-5,140-150$ & 55 & 61.8 & 0.45 & 8.04 & 3.07 & 1.64 & 0.87 & 1.86 \\
\hline $8-3,140-150$ & 63 & 51.9 & 0.53 & 10.52 & 4.20 & 2.04 & 5.96 & 2.28 \\
\hline $9-2,140-150$ & 72 & 53.5 & 0.43 & 7.81 & 3.88 & 1.81 & 8.24 & 2.42 \\
\hline $11-2,140-150$ & 90 & 67.7 & 0.30 & 5.55 & 2.36 & 1.14 & 0.65 & 1.26 \\
\hline $13-1,140-150$ & 110 & 62.5 & 0.37 & 6.51 & 2.91 & 1.26 & 3.76 & 1.64 \\
\hline $15-5,140-150$ & 130 & 65.0 & 0.36 & 7.46 & 2.87 & 1.50 & 1.71 & 1.74 \\
\hline $17-5,140-150$ & 149 & 66.0 & 0.45 & 10.42 & 3.15 & 1.18 & 0.87 & 2.16 \\
\hline $19-4,140-150$ & 170 & 59.4 & 0.51 & 11.91 & 3.98 & 1.55 & 3.22 & 2.47 \\
\hline $21-4,140-150$ & 188 & 64.9 & 0.44 & 9.59 & 3.40 & 1.39 & 0.96 & 1.96 \\
\hline $24-4,140-150$ & 212 & 63.4 & 0.46 & 10.41 & 2.96 & 1.42 & 1.76 & 1.91 \\
\hline $27-4,140-150$ & 245 & 60.5 & 0.47 & 10.61 & 3.34 & 1.58 & 2.30 & 2.01 \\
\hline $32-4,140-150$ & 283 & 61.1 & 0.54 & 11.46 & 3.47 & 1.41 & 1.02 & 2.61 \\
\hline $36-4,140-150$ & 330 & 63.3 & 0.49 & 11.83 & 3.37 & 1.52 & 0.95 & 2.34 \\
\hline $39-4,140-150$ & 360 & 64.8 & 0.51 & 10.03 & 3.12 & 1.30 & 1.00 & 1.97 \\
\hline $43-1,140-150$ & 395 & 56.3 & 0.50 & 11.50 & 3.62 & 2.63 & 5.14 & 2.30 \\
\hline $44-4,140-150$ & 409 & 50.1 & 0.45 & 10.24 & 3.62 & 4.82 & 7.92 & 1.93 \\
\hline $47-4,140-150$ & 438 & 55.3 & 0.58 & 15.44 & 4.59 & 2.81 & 4.86 & 3.12 \\
\hline
\end{tabular}

\section{Site 476 (Fig. 6)}

At this site sedimentation rates have averaged $\sim 42$ m/m.y. (Fig. 2), rates similar to those observed at Site 475 .

Again a distinct minimum in dissolved sulfate is observed in the upper sediment column, with almost complete depletion of sulfate at about 50 meters $(<2 \mathrm{mM})$. At the bottom of the sediment column, just above the unit containing metamorphic cobbles and illitic clays, dissolved sulfate concentrations are equal to normal sea-water concentrations. This poses an interesting problem: It is almost certain that even in the lower sediment section, below 66 meters sub-bottom, sulfate reduction processes have been operative. If this is so, it is difficult to understand why dissolved sulfate values at the bot- tom of the sediment section would be like those in sea water. There is little doubt that the sulfate minimum is induced by the relative increase in importance of sulfate reduction in the upper sediment column. However, the concentrations at the bottom of the sediment column do suggest that perhaps sea water penetrates advectively through the cobble zone, which may outcrop at the edges of the sediment terrace. If the latter is indeed the case, of course, the penetrating sea water must have interacted with the sediments or cobbles to cause a slight increase in dissolved calcium, a decrease in magnesium, and a decrease in the ${ }^{87} \mathrm{Sr} /{ }^{86} \mathrm{Sr}$ ratio of the dissolved strontium. This, of course, could also explain the sulfate minimum at Site 475 . Though the possibility of seawater penetration is intriguing, we cannot actually prove it. However, a similar penetration of sea water through 
Table 5. Bulk sediment atomic elemental ratios to aluminum, Sites 474,477 , and 479 .

\begin{tabular}{|c|c|c|c|c|c|}
\hline $\begin{array}{c}\text { Sample } \\
\text { (interval in } \mathrm{cm} \text { ) }\end{array}$ & $\mathrm{Si} / \mathrm{Al}$ & $\mathrm{Ti} / \mathrm{Al}$ & $\mathrm{Fe} / \mathrm{Al}$ & $\mathrm{Mg} / \mathrm{Al}$ & $\mathrm{K} / \mathrm{Al}$ \\
\hline \multicolumn{6}{|l|}{ Hole 474} \\
\hline $3-2,140-150$ & 3.39 & 0.025 & 0.225 & 0.181 & 0.246 \\
\hline $16-2,95-106$ & 3.11 & 0.027 & 0.252 & 0.229 & 0.228 \\
\hline \multicolumn{6}{|l|}{ Hole 474A } \\
\hline $4-5,140-150$ & 3.04 & 0.027 & 0.224 & 0.196 & 0.234 \\
\hline $16-4,14 c$ & 3.12 & 0.027 & 0.226 & 0.203 & 0.327 \\
\hline $23-3,140-150$ & 3.07 & 0.026 & 0.197 & 0.167 & 0.243 \\
\hline $30-2,140-150$ & 3.46 & 0.026 & 0.222 & 0.182 & 0.247 \\
\hline $39-1,140-150$ & 3.11 & 0.026 & 0.217 & 0.252 & 0.295 \\
\hline \multicolumn{6}{|l|}{ Hole 477} \\
\hline $2-3,140-150$ & 3.59 & 0.037 & 0.242 & 0.261 & 0.230 \\
\hline $3-1,140-1$ & 3.71 & 0.039 & 0.226 & 0.230 & 0.260 \\
\hline $4-1,133-140$ & 4.86 & 0.031 & 0.228 & 0.316 & 0.129 \\
\hline $5-1,140-150$ & 4.46 & 0.035 & 0.239 & 0.257 & 0.186 \\
\hline $7-1,142-150$ & 4.04 & 0.031 & 0.259 & 0.447 & 0.136 \\
\hline $7-2,27-32$ & 3.16 & 0.037 & 0.238 & 0.600 & 0.092 \\
\hline 9-1, Piece 6 & 3.41 & 0.089 & 0.500 & 0.630 & 0.007 \\
\hline $15-1,126-131$ & 2.38 & 0.032 & 0.245 & 0.963 & 0.252 \\
\hline $16-4,140-150$ & 3.78 & 0.032 & 0.411 & - & 0.179 \\
\hline $17-2,140-150$ & 3.72 & 0.034 & 0.229 & 0.301 & 0.318 \\
\hline $19-1,140-150$ & 3.90 & 0.039 & 0.207 & & 0.244 \\
\hline $20-1,140-150$ & 3.25 & 0.029 & 0.216 & 9 & 0.250 \\
\hline $22-1,140-150$ & 3.50 & 0.031 & 0.252 & 0.253 & 0.171 \\
\hline $23, \mathrm{CC}$ & 3.42 & 0.033 & 0.273 & 0.232 & 0.236 \\
\hline \multicolumn{6}{|l|}{ Hole 477A } \\
\hline $5-$ & 3.73 & .034 & 0.229 & 0.263 & 0.031 \\
\hline $6, \mathrm{CC}$ & 1.98 & 0.033 & 0.642 & - & 0.025 \\
\hline $7-1,3-7$ & 3.98 & 0.032 & 0.172 & 0.119 & 0.027 \\
\hline $8, \mathrm{CC}$ & 4.21 & 0.028 & 0.306 & 0.326 & 0.024 \\
\hline $9-1,69-7$ & 3.71 & 0.033 & 0.164 & 0.174 & 0.021 \\
\hline $10-1,110-120$ & 4.01 & 0.033 & 0.224 & 0.248 & 0.023 \\
\hline \multicolumn{6}{|l|}{ Hole 479} \\
\hline $1-1$, & 7.77 & 0.0 & 0.284 & 0.4 & 0.260 \\
\hline $3-1$ & 4.27 & 0 & 0.245 & 0. & 0.225 \\
\hline $5-3,110-120$ & 5.57 & 0.039 & 0.278 & 0.310 & 0.255 \\
\hline $7-5,140-150$ & 6.52 & 0.036 & 0.244 & 0.258 & 0.250 \\
\hline $8-3,140-150$ & 4.18 & 0.032 & 0.255 & 0.245 & 0.235 \\
\hline $9-2,140-1$ & 5.81 & 0.035 & 0.317 & 0.293 & 0.335 \\
\hline $11-2,140-150$ & 10.35 & 0.035 & 0.272 & 0.260 & 0.246 \\
\hline $13-1,140-150$ & 8.14 & 0.036 & 0.285 & 0.245 & 0.273 \\
\hline $15-5,140-1$ & 7.40 & 0.031 & 0.246 & 0.254 & 0.252 \\
\hline $17-5,140-150$ & 5.38 & 0.027 & 0.193 & 0.143 & 0.224 \\
\hline $19-4,140-150$ & 4.24 & 0.027 & 0.213 & 0.165 & 0.224 \\
\hline $21-4,140-150$ & 5.74 & 0.029 & 0.197 & 0.169 & 0.204 \\
\hline $24-4,140-150$ & 5.1 & 0.028 & 0.208 & 0.187 & 0.216 \\
\hline $27-4,140-150$ & 4.84 & 0.028 & 0.201 & 0.190 & 0.207 \\
\hline $32-4,140-150$ & 4.52 & 0.030 & 0.193 & 0.156 & 0.246 \\
\hline $36-4,140-150$ & 4.54 & 0.026 & 0.182 & 0.162 & 0.213 \\
\hline $39-4,140-150$ & 5.48 & 0.032 & 0.199 & 0.164 & 0.213 \\
\hline $43-1,140-150$ & 4.15 & 0.028 & 0.201 & 0.289 & 0.216 \\
\hline $44-1,140-150$ & 4.15 & 0.028 & 0.199 & 0.595 & 0.204 \\
\hline $47-4,140-150$ & 3.04 & 0.024 & 0.190 & 0.230 & 0.219 \\
\hline AVG-1 (Exp & 2.8 & 0.0 & 0.246 & 0.145 & \\
\hline AGV-1 (Flanagan) & 2.90 & 0.039 & 0.251 & 0.112 & \\
\hline
\end{tabular}

a metamorphic conglomerate was invoked by Gieskes and Johnson (1981b) for Site 453 in the Mariana Trough.

Of some interest is also the minimum in dissolved magnesium, which again may be induced by a relative
Table 6. Trace element chemistry in sediments, Sites 474,477 , and 481.

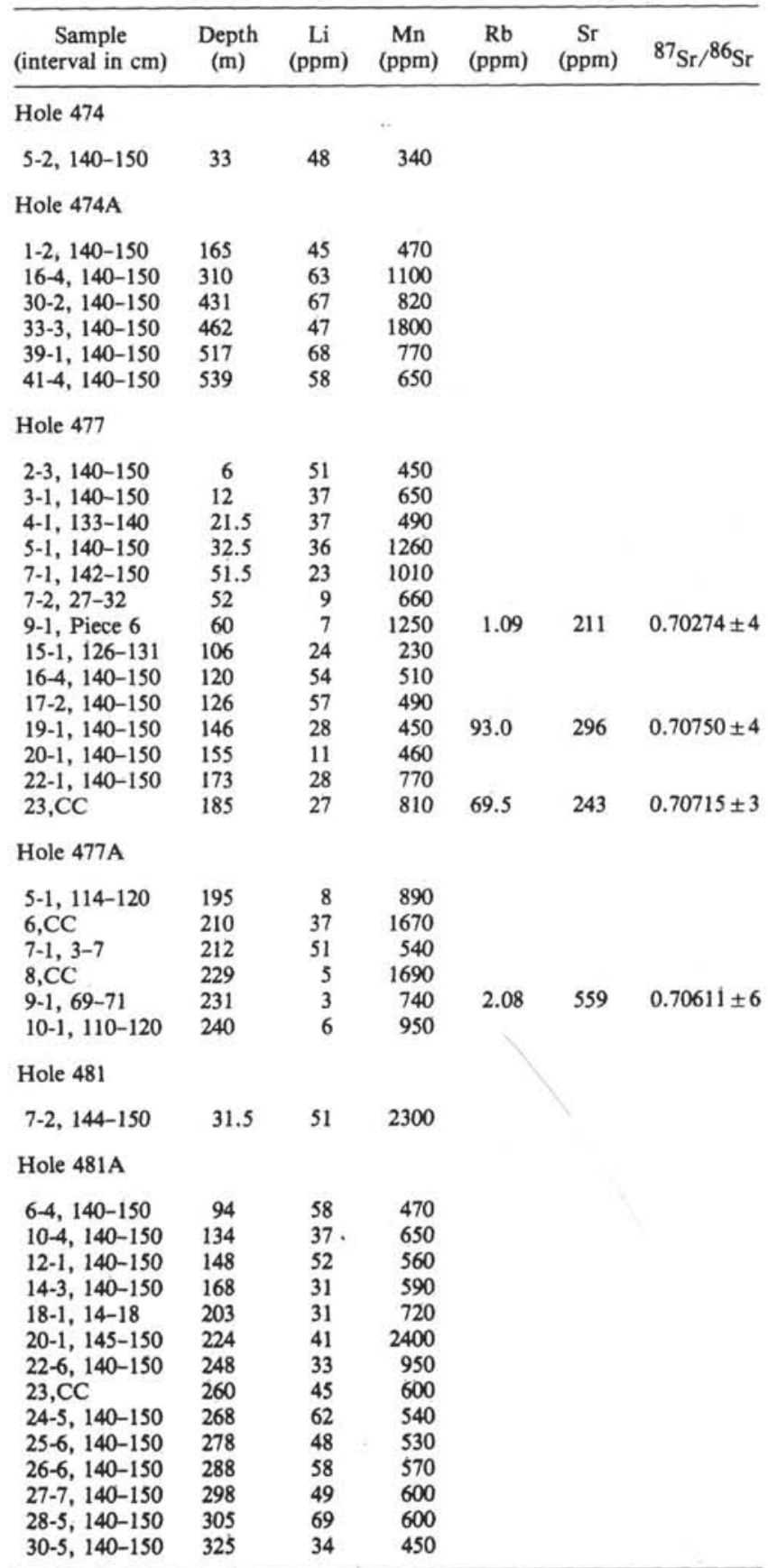

change in accumulation rates, or by the influx of sea water from below the sediment section.

\section{Site 477 (Figs. 7, 8, and 9)}

Site 477 is located very close to the heat flow anomaly first detected by Lawver et al. (1975). A temperature measurement at 48 meters sub-bottom indicates a temperature of $45^{\circ} \mathrm{C}$, for a temperature gradient of $\sim 88^{\circ} \mathrm{C}$ / $100 \mathrm{~m}$. The configuration of the heat probe used at that time would probably lead to an underestimate of the temperature, so that temperatures at the bottom of the hole must have exceeded $200^{\circ} \mathrm{C}$. 
Table 7. Protodolomite and dolomite samples, in wt.\%, Leg 64.

\begin{tabular}{|c|c|c|c|c|c|c|c|c|}
\hline $\begin{array}{c}\text { Sample } \\
\text { (interval in } \mathrm{cm} \text { ) }\end{array}$ & $\begin{array}{l}\text { Depth } \\
\text { (m) }\end{array}$ & $\mathrm{SiO}_{2}$ & $\mathrm{TiO}_{2}$ & $\mathrm{Al}_{2} \mathrm{O}_{3}$ & $\mathrm{Fe}_{2} \mathrm{O}_{3}$ & $\mathrm{MgO}$ & $\mathrm{CaO}$ & $\mathrm{K}_{2} \mathrm{O}$ \\
\hline \multicolumn{9}{|l|}{ Hole 479} \\
\hline $10, \mathrm{CC}, 12$ & 87.4 & 24.26 & 0.19 & 4.56 & 1.73 & 8.70 & 20.26 & 1.16 \\
\hline $10-6,92-94$ & 88.4 & 29.67 & 0.45 & 5.76 & 2.15 & 10.32 & 20.76 & 0.87 \\
\hline $13, \mathrm{CC}$ & 110.8 & 20.28 & 0.16 & 3.55 & 1.09 & 14.09 & 23.73 & 0.42 \\
\hline $23-1,58-59$ & 203.1 & 28.80 & 0.20 & 5.79 & 2.53 & 10.28 & 17.98 & 0.98 \\
\hline $27, \mathrm{CC}$ & 250 & 9.85 & 0.14 & 2.44 & 0.82 & 15.89 & 29.87 & 0.22 \\
\hline $29-1,1-4$ & 259.5 & 20.20 & 0.19 & 4.82 & 4.20 & 12.50 & 24.64 & 1.40 \\
\hline $29-1,13-14$ & 259.6 & 9.67 & 0.13 & 2.58 & 1.44 & 16.01 & 24.86 & 0.57 \\
\hline $29-1,26-26.5$ & 259.8 & 30.60 & 0.32 & 6.77 & 4.24 & 10.20 & 17.64 & 1.30 \\
\hline 32, CC "light" & 290.2 & 56.15 & 0.54 & 13.50 & 4.23 & & 1.39 & 2.49 \\
\hline 32,CC "dark" & 290.2 & 9.05 & 0.09 & 2.20 & 1.15 & 17.20 & 29.31 & 0.28 \\
\hline $34-5,148-149$ & 314.2 & 10.10 & 0.12 & 2.03 & 3.18 & 16.13 & 28.44 & 0.32 \\
\hline $41, \mathrm{CC}$ & 375 & 33.72 & 0.30 & 7.42 & 2.79 & 10.42 & 16.25 & 1.28 \\
\hline $44, C C$ & 411 & 19.59 & 0.20 & 5.45 & 5.14 & 12.20 & 23.84 & 0.72 \\
\hline $45, \mathrm{CC}$ & 414.6 & 13.42 & 0.19 & 4.64 & 8.37 & 12.56 & 27.13 & 0.72 \\
\hline $47-2,124-126$ & 433.2 & 10.37 & 0.14 & 3.57 & 2.75 & 17.61 & 27.64 & 0.40 \\
\hline $47-2,134-156$ & 433.3 & 11.18 & 0.14 & 3.88 & 2.20 & 17.89 & 26.97 & 0.51 \\
\hline $47-2,145-147$ & 433.4 & 10.96 & 0.14 & 3.82 & 2.97 & 17.09 & 29.52 & 0.50 \\
\hline \multicolumn{9}{|l|}{ Hole 478} \\
\hline $22, \mathrm{CC}$ & 188.3 & 19.71 & 0.18 & 4.66 & 2.14 & 14.70 & 25.06 & 0.76 \\
\hline \multicolumn{9}{|l|}{ Hole 480} \\
\hline 21,1 & 100 & 12.03 & 0.12 & 0.92 & 0.70 & 14.48 & 32.36 & 0.20 \\
\hline
\end{tabular}

A major phenomenon at this site is the occurrence of sills in an apparently cooled-off state in Holes 477 (58$105.5 \mathrm{~m})$ and $477 \mathrm{~A}(32.5-62.5 \mathrm{~m})$. The sills must be relatively old, probably 20,000 years or more, to have cooled off so completely (Vacquier, this volume, Pt. 2). With sedimentation rates estimated to be as high as $2000 \mathrm{~m} /$ m.y., the sediment thickness at the time of intrusion of the sills was probably 25 meters or less in this site. The heat source leading to the observed high heat flow must be located deeper in the site, as is also clear from the continuous increase in temperature with depth observed during the logging of this hole.

Interstitial water concentration profiles show great complexity at this site (Fig. 7). The upper 30 meters, which probably have been deposited after the cooling of the upper sills, indicate concentration changes typically observed in rapidly accumulating sediments with high organic carbon contents. A sharp increase in alkalinity is accompanied by a rapid lowering of dissolved sulfate, increases in ammonia and phosphate, a decrease in dissolved calcium, and finally a reversal in magnesium concentrations, observations similar to those made at Site 474.

Below 30 meters toward the sill in Hole 477 as well as just below the sill, the influence of the doleritic sill intrusion is clearly observable in the interstitial water concentrations. Alkalinity and dissolved ammonia values rapidly decrease and dissolved silica, at least directly below the sill, also appears strongly affected. Dissolved sulfate and dissolved magnesium appear especially influenced by the sill intrusion, with the dissolved sulfate concentrations being relatively high both above and below the sill. The latter increase is also accompanied by high magnesium concentrations. These observations can be explained in two ways: (1) relatively unaltered sea water has been trapped in the sill and is diffusing into the surrounding sediments, or (2) sulfates, precipitated during the hydrothermal interaction between the penetrating dolerite sills and sea water/pore water, are presently dissolving, and the resultant sulfate ions will diffuse into the sediments. We consider the first possibility

Table 8. Elemental atomic ratios to $\mathrm{Al}$ and calculated protodolomite composition ${ }^{\mathrm{a}}$ in protodolomite samples, Holes 479,480 , and 478 .

\begin{tabular}{|c|c|c|c|c|c|c|c|c|c|c|}
\hline $\begin{array}{c}\text { Sample } \\
\text { (interval in } \mathrm{cm} \text { ) }\end{array}$ & $\begin{array}{l}\text { Depth } \\
\text { (m) }\end{array}$ & $\mathrm{Ti} / \mathrm{Al}$ & $\mathrm{Fe} / \mathrm{Al}$ & $\mathrm{Ca} / \mathrm{Al}$ & $\mathrm{Mg} / \mathrm{Al}$ & $\mathrm{K} / \mathrm{Al}$ & $\mathrm{Si} / \mathrm{Al}$ & $\begin{array}{c}\mathrm{CaCO}_{3}{ }^{\circ} \\
(\%)\end{array}$ & $\begin{array}{c}\mathrm{MgCO}_{3} \\
(\%)\end{array}$ & $\begin{array}{c}\mathrm{FeCO}_{3} \\
(\%)\end{array}$ \\
\hline \multicolumn{11}{|l|}{ Hole 479} \\
\hline $10-6,92-94$ & 87.4 & 0.050 & 0.238 & 3.2 & 1.9 & 0.218 & 4.37 & 64 & 36 & \\
\hline $10, \mathrm{CC}, 12$ & 88.4 & 0.027 & 0.242 & 4.1 & 2.9 & 0.206 & 4.51 & 59 & 41 & \\
\hline $13, \mathrm{CC}$ & 110.8 & 0.029 & 0.196 & 6.1 & 5.0 & 0.128 & 4.85 & 55 & 45 & \\
\hline $23-1,58-59$ & 203.1 & 0.022 & 0.279 & 2.8 & 2.2 & 0.183 & 4.22 & 56 & 42 & 2 \\
\hline $27, \mathrm{CC}$ & 250 & 0.037 & 0.215 & 11.1 & 8.2 & 0.098 & 3.42 & 58 & 42 & 0 \\
\hline $29-1,1.5-4$ & 259.5 & 0.025 & 0.556 & 4.6 & 3.3 & 0.314 & 3.56 & 56 & 39 & 4.5 \\
\hline $29-1,13-14$ & 259.6 & 0.032 & 0.356 & 8.8 & 7.8 & 0.239 & 3.18 & 53 & 46 & 1 \\
\hline $29-1,26-26.5$ & 259.8 & 0.030 & 0.400 & 2.4 & 1.9 & 0.208 & 3.84 & 55 & 40 & 5 \\
\hline $32, \mathrm{CC}$ (dark) & 290.2 & 0.026 & 0.334 & 12.1 & 9.9 & 0.138 & 3.49 & 55 & 44 & 0.6 \\
\hline $32, \mathrm{CC}$ (light) & 290.2 & 0.026 & 0.200 & 0.09 & & 0.200 & 3.53 & & & \\
\hline $34-5,148-149$ & 314.2 & 0.038 & 1.00 & 12.7 & 10.0 & 0.171 & 4.22 & 54 & 42 & 3 \\
\hline $41, \mathrm{CC}$ & 375 & 0.026 & 0.240 & 2.0 & 1.8 & 0.187 & 3.86 & 54 & 45 & 1 \\
\hline $44, \mathrm{CC}$ & 411 & 0.023 & 0.602 & 4.0 & 2.8 & 0.143 & 3.05 & 56 & 38 & 6 \\
\hline $45, \mathrm{CC}$ & 414.6 & 0.026 & 1.15 & 5.3 & 3.4 & 0.168 & 2.45 & 56 & 34 & 10 \\
\hline $47-2,124-126$ & 433.2 & 0.025 & 0.492 & 7.0 & 6.2 & 0.121 & 2.46 & 52 & 46 & 2 \\
\hline $47-2,134-136$ & 433.3 & 0.023 & 0.362 & 6.3 & 5.8 & 0.142 & 2.44 & 52 & 47 & 1 \\
\hline $47-2,145-147$ & 433.4 & 0.023 & 0.496 & 7.0 & 5.7 & 0.142 & 2.43 & 54 & 43 & 2 \\
\hline \multicolumn{11}{|l|}{ Hole 480} \\
\hline $21-1$ & 100 & 0.083 & 0.486 & 32.0 & 19.9 & 0.235 & 11.10 & & & \\
\hline \multicolumn{11}{|l|}{ Hole 478} \\
\hline $22, \mathrm{CC}$ & 188.3 & 0.025 & 0.293 & 4.9 & 4.0 & 0.176 & 3.49 & & & \\
\hline
\end{tabular}

a The composition of the protodolomites was calculated as follows: The $\mathrm{Ca} / \mathrm{Al}, \mathrm{Mg} / \mathrm{Al}$, and $\mathrm{Fe} / \mathrm{Al}$ ratios were corrected for background values calculated for the nondolomite sediments $(\mathrm{Ca} / \mathrm{Al}=0.4$ upper $150 \mathrm{~m}, \mathrm{Ca} / \mathrm{Al}$ $=0.1$ below $150 \mathrm{~m} ; \mathrm{Mg} / \mathrm{Al}=0.3$ upper $150 \mathrm{~m}, \mathrm{Mg} / \mathrm{Al}=0.2$ below $150 \mathrm{~m} ; \mathrm{Fe} / \mathrm{Al}=0.2$ below $150 \mathrm{~m}$ ). The percentage of $\mathrm{CaCO}_{3}, \mathrm{MgCO}_{3}$, and $\mathrm{FeCO}_{3}$ was then calculated from the corrected ratios. It was assumed that all other constituents of the protodolomites were $<1 \%$.

The estimated errors were $\pm 3 \%$ of the $\mathrm{CaCO}_{3}$ value, $\pm 4 \%$ of the $\mathrm{MgCO}_{3}$ value, and $\pm 10 \%$ of the $\mathrm{FeCO}$ value. These estimated errors were obtained by taking a low and a high correction for $\mathrm{Ca} / \mathrm{Al}, \mathrm{Mg} / \mathrm{Al}$, and $\mathrm{Fe} / \mathrm{Al}$ and calculating the spread for the corrected values of several samples containing high Al. 


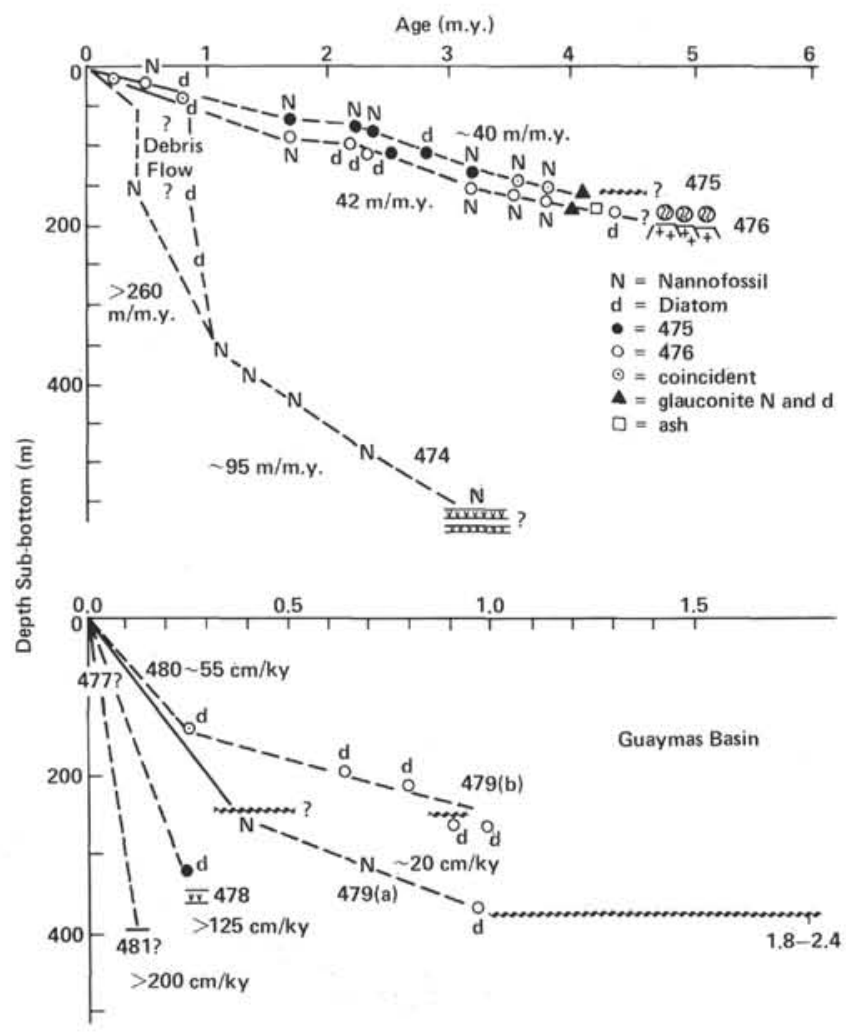

Figure 2. Sedimentation rate diagram, Leg 64. rather improbable and prefer the second. Edmond et al. (1979) and Bischoff and Seyfried (1978) indicate that a magnesium-hydroxy sulfate forms rapidly during hydrothermal interaction between sea water and basalts, or by heating sea water itself. It is, therefore, possible that small quantities of this material (as well as gypsum and anhydrite) have formed during the penetration of the sills. Quantitatively these minerals may be unimportant but would nevertheless leave an imprint on the pore fluids. No magnesium-hydroxy sulfate has been detected (M. Kastner, pers. comm.), but the dissolution of such material would explain the observed magnesium and sulfate anomalies most satisfactorily. No precise stoichiometry would be expected because of the different reactivities of sulfate and magnesium in the basalt-pore-water-sediment system. Indeed the dolerite sill appears to serve as a sink for ammonia, potassium, and perhaps magnesium as a result of relatively cold temperature alteration reactions.

Below 140 meters the influence of hydrothermal reactions taking place in the sediments and underlying basalts(?) leaves a clear imprint on the interstitial waters. Typically sulfate becomes depleted and magnesium disappears quantitatively from the interstitial waters. Increases in dissolved calcium, lithium, potassium, rubidium, and ammonia are evident, much in accord with observations made on hydrothermal fluids emanating from ridge crests (e.g., Edmond et al., 1979). A quanti-
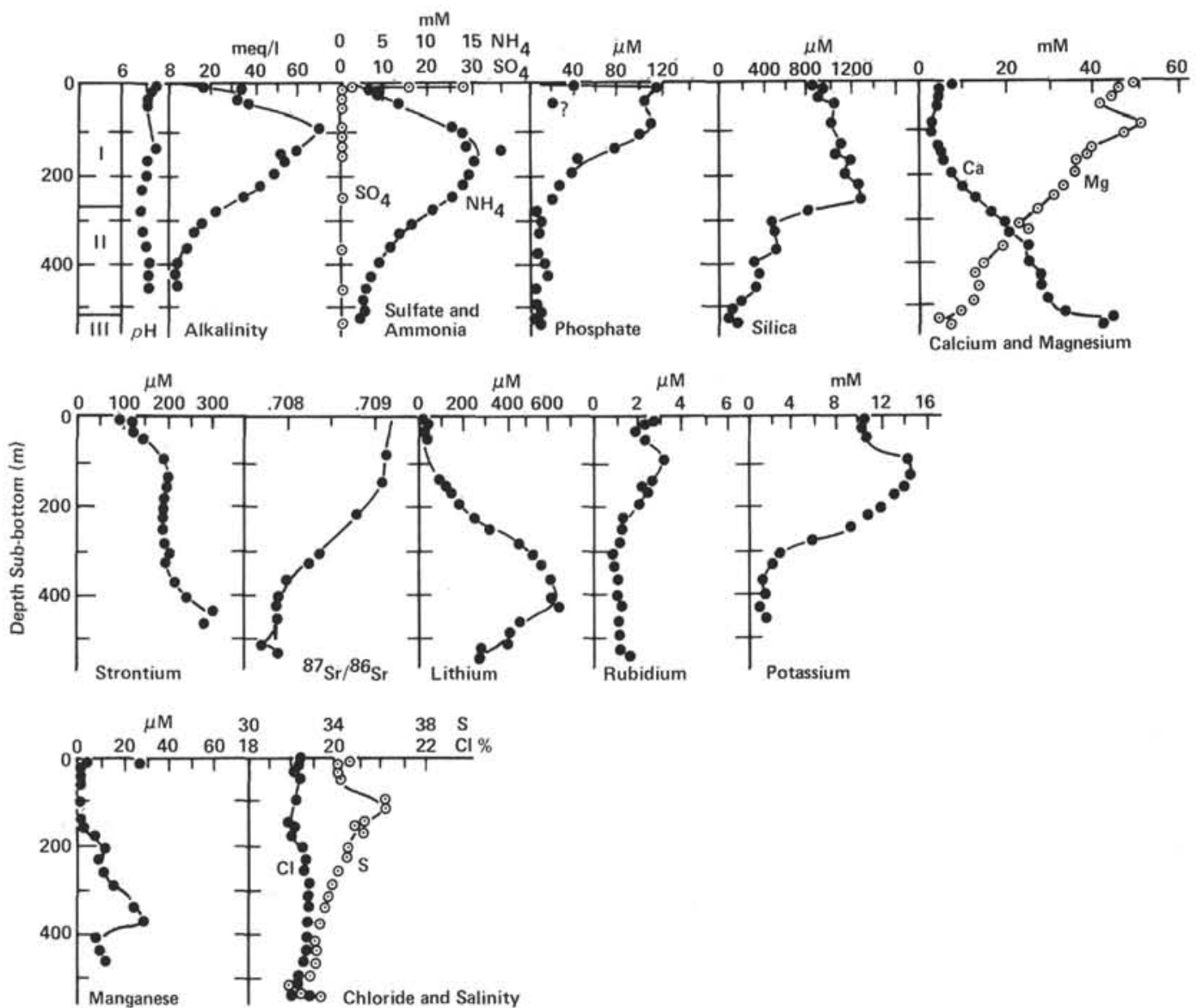

Figure 3. Interstitial water chemistry, Site 474. Lithology: I, diatomaceous oozes, muds, and turbidites; II, silty claystone and clayey siltstone-graded mud turbidites; III, dolerite sills and claystones. 


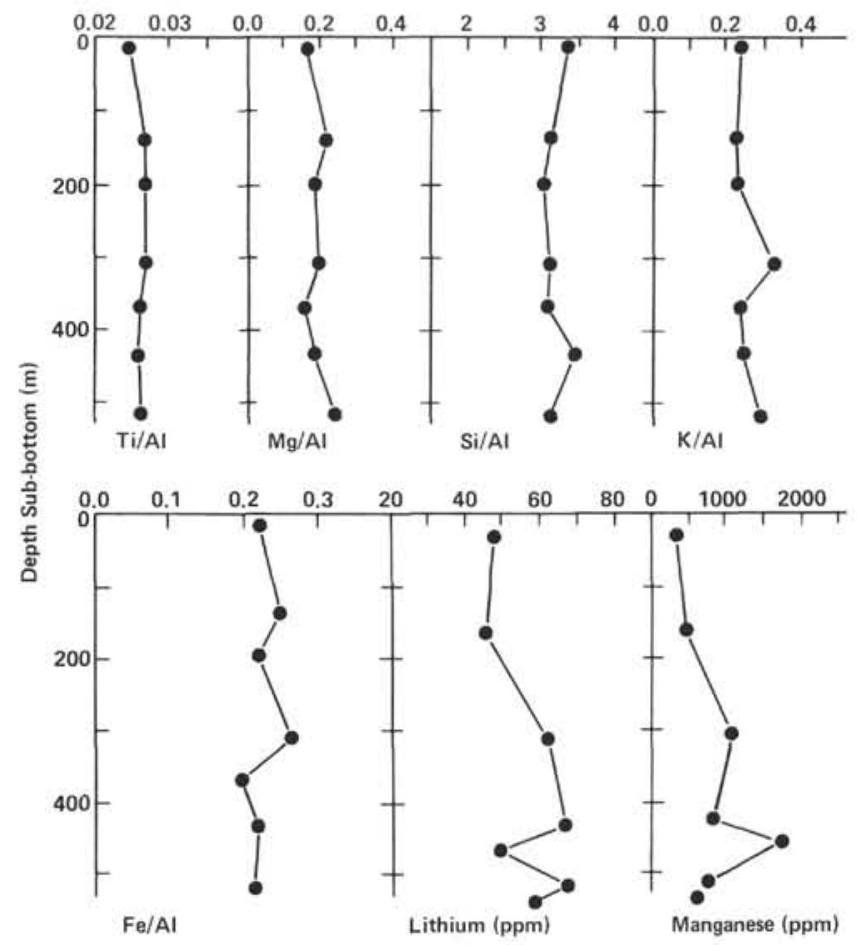

Figure 4. Elemental atomic ratios to aluminum of bulk sediment at Site 474; $\mathrm{Li}$ and $\mathrm{Mn}$ in concentrations (ppm).

tative comparison, however, is not possible because our samples were obtained under conditions different from those prevailing in situ. The concentrations for $\mathrm{K}^{+}$, $\mathrm{Li}^{+}, \mathrm{Rb}^{+}$and $\mathrm{NH}_{4}{ }^{+}$may be underestimates, especially in view of the well-known temperature-of-squeezing effects (Sayles and Manheim, 1975). Among these effects, that on $\mathrm{K}^{+}$is probably the best documented: an increase
Table 9. $\delta^{18} \mathrm{O}$ and $\delta^{13} \mathrm{C}$ of calcites in basalts at Site 474.

\begin{tabular}{lccccr}
\hline $\begin{array}{c}\text { Sample } \\
\text { (interval in cm) }\end{array}$ & $\begin{array}{c}\text { Depth } \\
(\mathrm{m})\end{array}$ & $\begin{array}{c}\delta^{18} \mathrm{O}(\mathrm{PDB}) \\
(\%)\end{array}$ & $\begin{array}{c}\delta^{13} \mathrm{C}(\mathrm{PDB}) \\
(\%)\end{array}$ & $\begin{array}{c}\delta^{18} \mathrm{O}(\mathrm{SMOW}) \\
(\%)\end{array}$ & $\begin{array}{c}\text { Temp. } \\
\left({ }^{\circ} \mathrm{C}\right)\end{array}$ \\
\hline $474 \mathrm{~A}-40-2,55-57$ & 526.56 & -4.16 & -19.84 & 26.57 & 34.0 \\
$474 \mathrm{~A}-41-5,129-133$ & 541.31 & -5.23 & -27.37 & 25.47 & 40.0 \\
$474 \mathrm{~A}-42-5,10-12$ & 549.61 & 2.72 & 0.30 & 33.89 & 1.8 \\
$474 \mathrm{~A}-44-5,112-114$ & 569.63 & 1.17 & 0.36 & 32.07 & 8.9 \\
\hline
\end{tabular}

in $\mathrm{K}^{+}$concentrations of $15-20 \%$ has been estimated for a $20^{\circ} \mathrm{C}$ rise in temperature $\left(4-24^{\circ} \mathrm{C}\right)$. This temperature effect appears confirmed from our only in situ sample, which indicates an effect of $30 \%$ for a $40^{\circ} \mathrm{C}$ temperature change (Table 2). This implies that $\mathrm{K}^{+}$concentrations in situ may be as high as $125 \mathrm{mM}$ in the deeper parts of this site. Clearly it is unfortunate that no in situ samples could be obtained in these deeper parts. In addition since sample sizes were small and sea-water contamination always present, a meaningful temperatureof-squeezing program would have been very difficult to perform. Thus, though our observations are necessarily qualitative, there is little doubt that large increases in the concentrations of the alkali metals result from hydrothermal interactions between sediments or basalts. These changes are larger than those estimated by Edmond et al. (1979), but, of course, the parent sediments in the Guaymas Basin are much more enriched in $\mathrm{Li}^{+}$, $\mathrm{K}^{+}$, and $\mathrm{Rb}^{+}$than are ridge crest basalts. This, for example, is clear from the data in Tables 4-6. Indeed, the concentrations of $\mathrm{K}^{+}$in the solids (Table 4, Fig. 9) show that the $\mathrm{K}_{2} \mathrm{O}$ is almost quantitatively removed from the sediments in Hole 477A.

The increase in dissolved chloride is about $15 \%$; it indicates a substantial removal of water, presumably as water of hydration, during the formation of hydrothermal minerals.

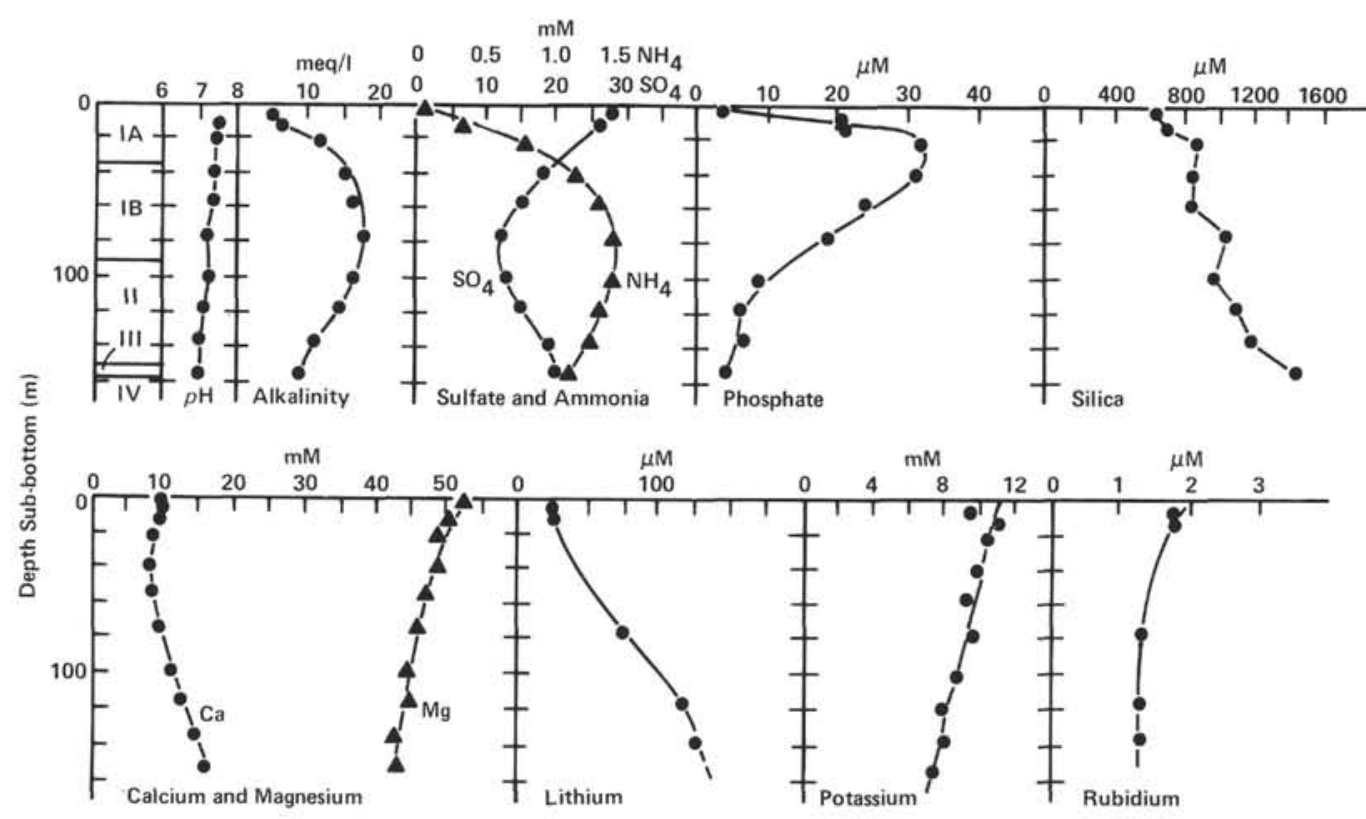

Figure 5. Interstitial water chemistry, Site 475. Lithology: IA, nannofossil diatomaceous silty clay to clayey silt; IB, clayey silt to silty clay; II, diatomaceous silty clay; III, zeolite-bearing clay, dolomitic mudstone; IV, metavolcanic conglomerate. 


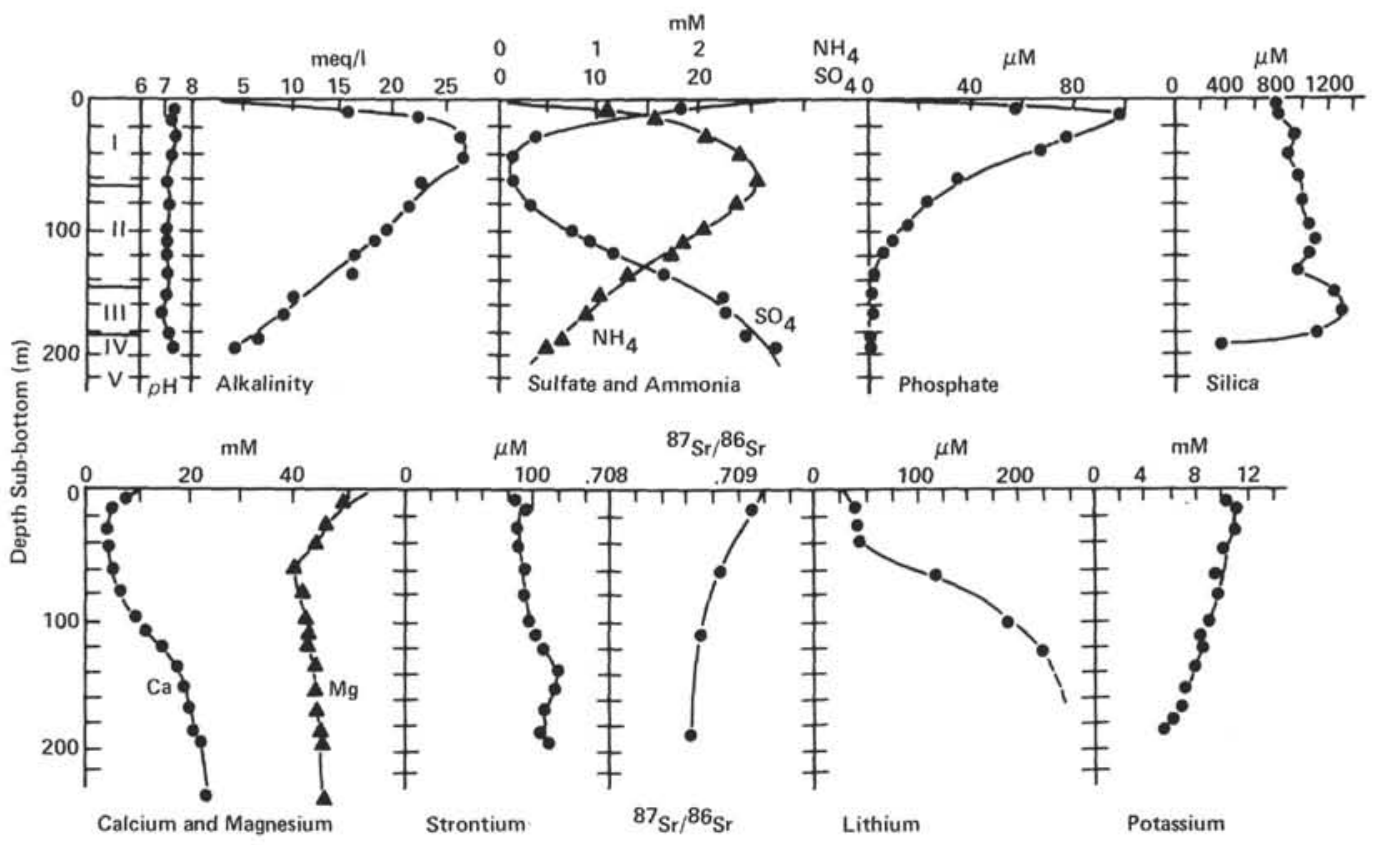

Figure 6. Interstitial water chemistry, Site 476. Lithology: I, nannofossil and diatomaceous oozes to muds; II, silty clay; III, muddy diatomaceous oozes, turbidites, glauconitic sands; IV, glauconite sands, zeolitic silty clays, phosphorite, pyritic organic claystone; $\mathrm{V}$, metamorphic cobbles and sandy clay.

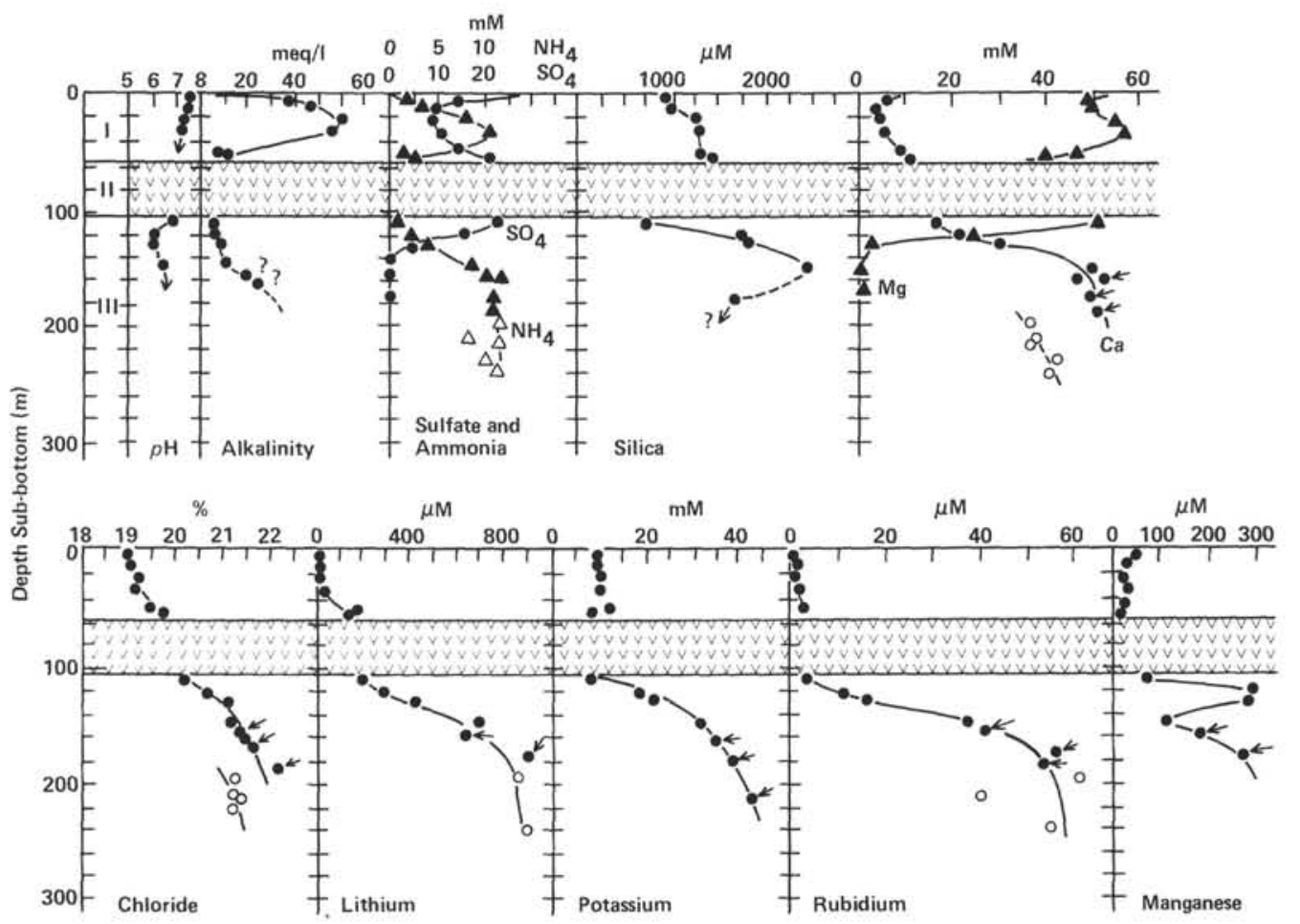

Figure 7. Interstitial water chemistry, Site 477. Lithology: I, diatomaceous ooze, sandy silts, and silty clay (turbidites); II, dolerite sill; III, hydrothermally altered clay and claystone, sandy siltstone. (Data with arrows are corrected for sea-water contamination. Open symbols are corrected data for Hole 477A.)

Of importance in this site are the results of the studies of the oxygen and strontium isotopes of the waters and dissolved strontium. The $\delta^{18} \mathrm{O}$ of the interstitial waters below the sill increases to values as high as $+1.37 \%$ ( $\mathrm{M}$. Kastner, pers. comm.). The values of $\delta^{18} \mathrm{O}$ of the bulk solid silicates show a gradual decrease (Kastner, this vol- ume, Pt. 2), testifying to the importance of hydrothermal alteration of the sediments in this site. The data obtained for the ${ }^{87} \mathrm{Sr} /{ }^{86} \mathrm{Sr}$ ratio in the interstitial waters, as well as for some selected solids, are presented in Figure 8. Though the lowermost two of the interstitial-water ${ }^{87} \mathrm{Sr} /{ }^{86} \mathrm{Sr}$ values have been corrected for sea-water con- 


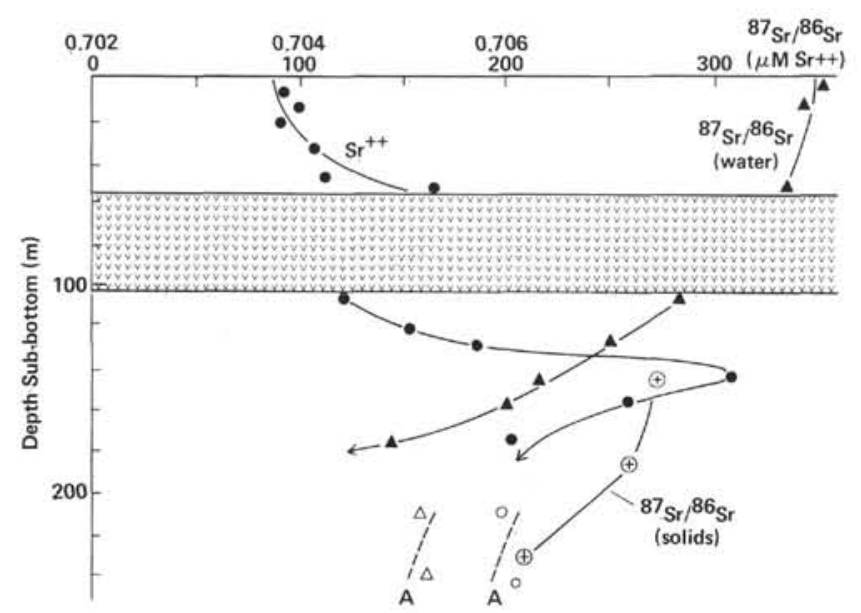

Figure 8. Dissolved strontium and ${ }^{87} \mathrm{Sr} /{ }^{86} \mathrm{Sr}$ of interstitial water strontium and solid phases, Site 477. Open circles: $\mathrm{Sr}^{++}$in 477A; open triangles: ${ }^{87} \mathrm{Sr} /{ }^{86} \mathrm{Sr}$ in $477 \mathrm{~A}$.

tamination in a manner similar to that described in the methods section, it is evident that very low values are reached in Holes 477 and 477A. The interstitial water values are lower than those in the bulk solids. This can be interpreted in two ways: (1) the sediments, consisting mainly of detrital material of volcanic origin and of biogenic silica, lose their strontium preferentially from their volcanic sources during hydrothermal alteration, or (2) a large basaltic imprint on the strontium isotopic composition of the dissolved strontium occurs. We propose to perform leaching experiments to determine whether the first hypothesis is viable, but at present we prefer the hypothesis of a basalt $\mathrm{Sr}$-isotope signal. John Lupton (pers. comm.) has, indeed, shown excess $\delta^{3} \mathrm{He}$ values in cap gases in this site $\left({ }^{3} \mathrm{He} /{ }^{4} \mathrm{He}\right.$ [sample/air] $=6.3$ at 144 $\mathrm{m})$. Such values elsewhere have been shown to reflect hydrothermal processes (Lupton, 1979). Furthermore the ${ }^{87} \mathrm{Sr} /{ }^{86} \mathrm{Sr}$ results are similar to those found by Albarede et al. (1980) in hydrothermal waters of the East Pacific Rise at $21^{\circ} \mathrm{N}$ as well as geothermal sea-water systems in Iceland, where Sr-isotope exchange has led to very low values in the circulating sea water (Elderfield and Greaves, pers. comm.). Of some interest here is the dissolved strontium profile, which shows a maximum at about 140 meters depth. This may indicate that strontium is removed from the pore fluids in the deeper parts of the site. Humphris and Thompson (1978) also describe strontium enrichments in epidotes, indicative of basalt-sea-water alteration at low water/rock ratios (Menzies and Seyfried, 1979). If this is indeed the case, a basaltic contribution to the dissolved ${ }^{87} \mathrm{Sr} /{ }^{86} \mathrm{Sr}$ profiles seems evident, and our data may be part of a general phenomenon.

The analysis of the bulk chemical composition of the solid phases (Tables 4 and 6, Fig. 9) clearly indicates the influence of the sill intrusion on the surrounding sediments (elevated $\mathrm{Mg} / \mathrm{Al}$, depressed $\mathrm{Si} / \mathrm{Al}, \mathrm{K} / \mathrm{Al}$, and lowered $\mathrm{Li}$ concentrations). In the deeper parts of the site, especially in Hole 477A, the influence of hydrothermal alteration is mostly evident from the very low $\mathrm{K} / \mathrm{Al}$ in the bulk solids below 190 meters. Ratios of $\mathrm{Mg} / \mathrm{Al}$,

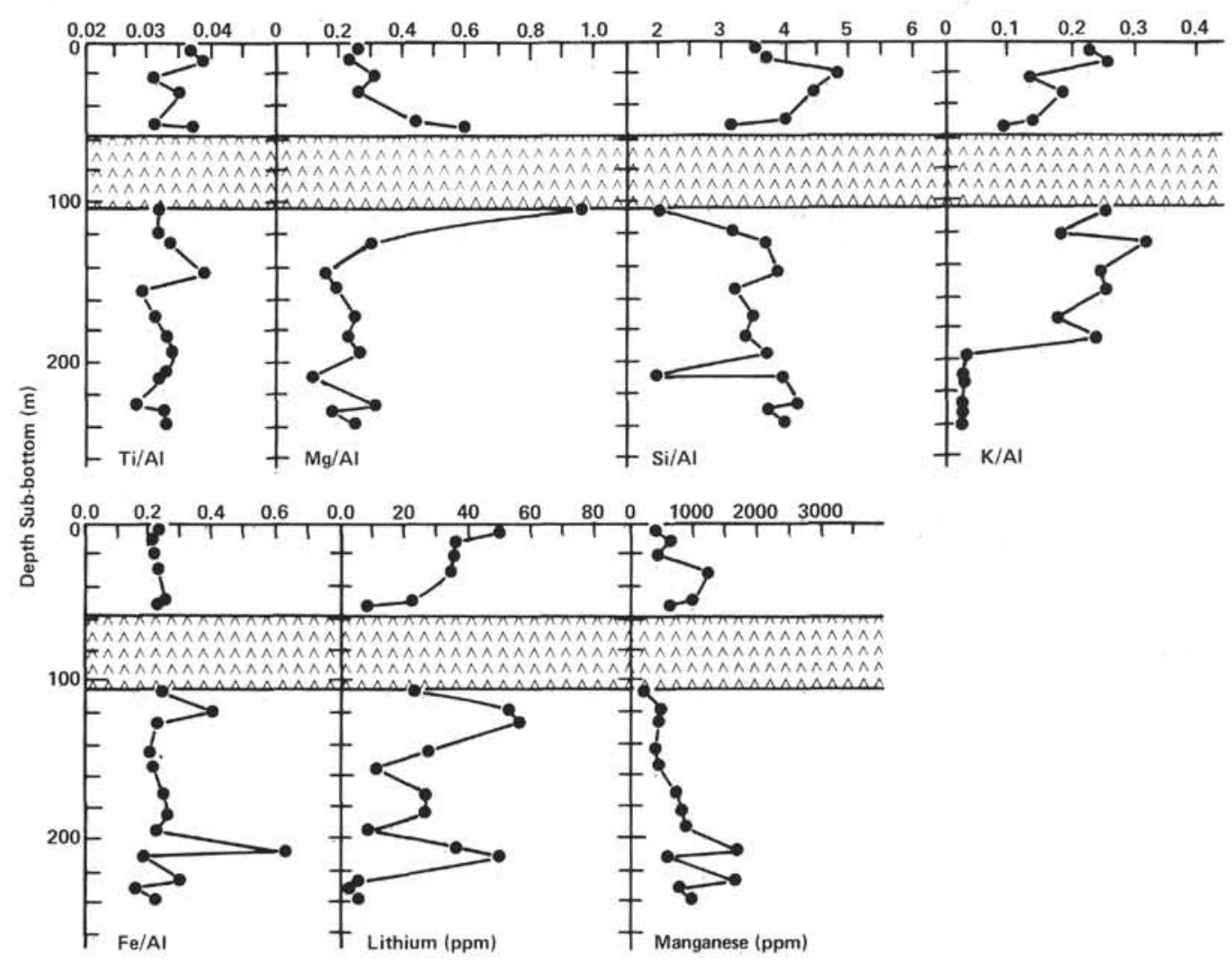

Figure 9. Elemental atomic ratios to aluminum of sediments at Site 477. Li and $\mathrm{Mn}$ in concentrations (ppm). 
$\mathrm{Si} / \mathrm{Al}$, and $\mathrm{Fe} / \mathrm{Al}$ appear little affected. The lack of increase in the $\mathrm{Mg} / \mathrm{Al}$ ratio is probably the result of complete removal of $\mathrm{Mg}^{++}$from the pore fluids prior to reaching the zones of alteration in this site. Only those elements that are efficiently removed during hydrothermal interaction $-\mathrm{K}^{+}$and $\mathrm{Li}^{+}$-would show depletions in the solids; here this is clearly the case, especially for $\mathrm{K}^{+}$.

\section{Site 478 (Fig. 10)}

Interstitial water profiles obtained in this site show great complexity. Concentration-depth profiles of alkalinity, sulfate, ammonia, phosphate, and calcium all show breaks at about 60 meters sub-bottom depth. Ammonia production between $60-170$ meters again appears to induce a maximum in dissolved magnesium and perhaps in potassium. All these data imply non-steady-state conditions at this site, with biogenic activity involving the degradation of organic matter being more important below than above 60 meters. Whether this situation is a result of differential sedimentation rates, of different reactivities of organic carbon, of the deposition of large sections of turbidites with lesser bacterial activity, or of a combination of these factors cannot be determined at this stage. It is clear, however, that as a result of the very high accumulation rates concentration changes occur in the interstitial waters that reflect non-steady-state conditions.

Within 20 meters above and below the limestonechert-dolerite complex between 190-230 meters, the influence of this complex is noticeable in the pore fluids. For instance, a rapid change occurs in dissolved magnesium, potassium, strontium (below sill), and lithium.
This appears to be related mostly to the sill intrusions, which must have had an influence on the surrounding sediments during the penetration at high temperatures and subsequently may have undergone low-temperature alteration reactions. The latter would lead to the uptake of magnesium (though magnesium may also be consumed during dolomitization of the limestones) and potassium. Below the sills the ${ }^{87} \mathrm{Sr} / 86 \mathrm{Sr}$ ratio of dissolved strontium is significantly lower than above the sills. Dissolved lithium shows a more complex distribution, especially below the sill complex. Below the sills there occurs a maximum in $\mathrm{Li}^{+}$which may be explained best in terms of a decaying dissolved-lithium profile: during the high-temperature stage dissolved lithium values may have been very high, but since that period uptake reactions during low-temperature alteration of the sills and diffusion into the overlying and underlying sediments have led to the gradual disappearance of the very high lithium concentrations. Measurements of the ${ }^{18} \mathrm{O} /$ ${ }^{16} \mathrm{O}$ composition of interstitial waters obtained below the sill indicate values that may be slightly higher in $\delta^{18} \mathrm{O}$ than sea water is (M. Kastner, pers. comm.).

A clear minimum in dissolved chloride occurs at about 80 meters sub-bottom. Below this minimum the chlorinities increase to slightly higher than present-day bottom water values $(19.3 \%$ o chlorinity). The occurrence of the chlorinity minimum will be discussed for Site 479, where a more pronounced minimum has been observed.

\section{Site 479 (Figs. 11 and 12)}

Site 479 is located on the slope of the Guaymas Basin at a water depth of 747 meters. The sediment accumulation rates are rapid in the upper section of this site

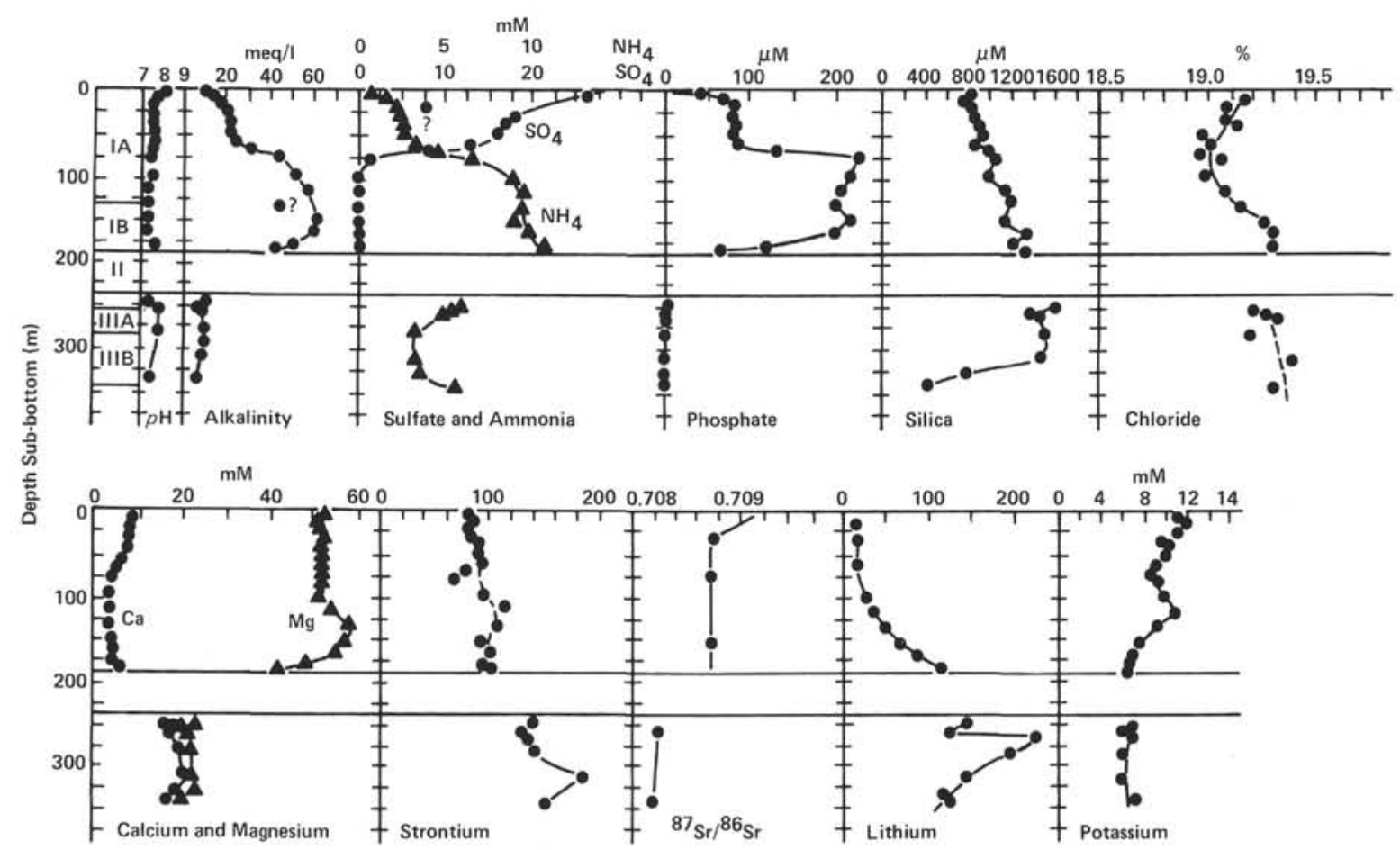

Figure 10. Interstitial water chemistry, Site 478. Lithology: IA, diatomaceous ooze with frequent turbidites; IB, diatomaceous silty mud; II, dolomitic siltstones, two dolerite sills, diatomaceous mudstone; IIIA, diatomaceous mudstone, laminated diatomaceous mudstone with dolomite; IIIB, siltstone and contact zone to basaltic intrusion. 


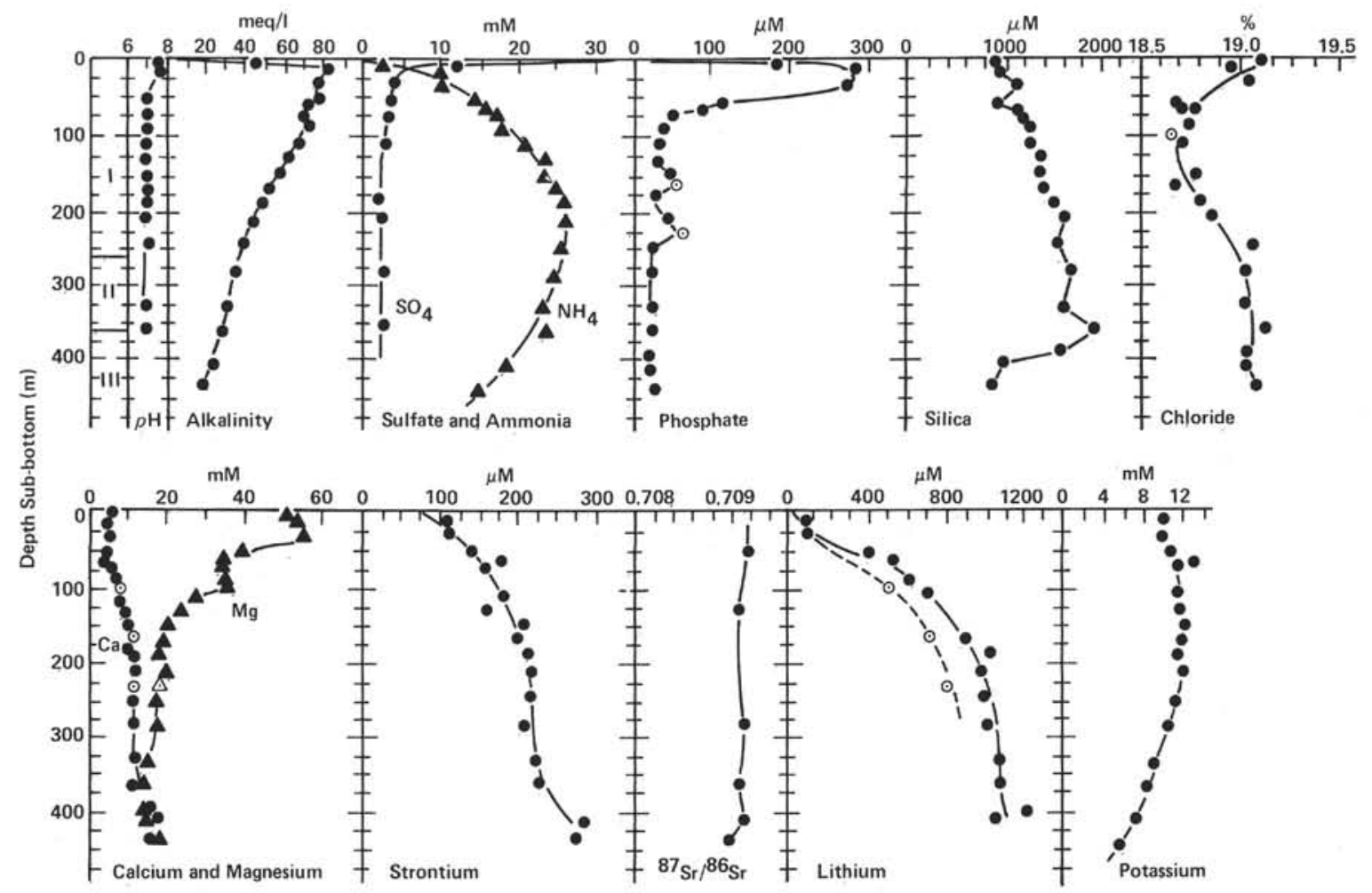

Figure 11. Interstitial water chemistry, Site 479. Lithology: I, soft unconsolidated diatomaceous oozes and muds, 4 calcareous limestone (dolomitic) beds; II, increased induration; limestone beds; III, claystones; limestone beds. (Open circles and triangles are in situ data.)

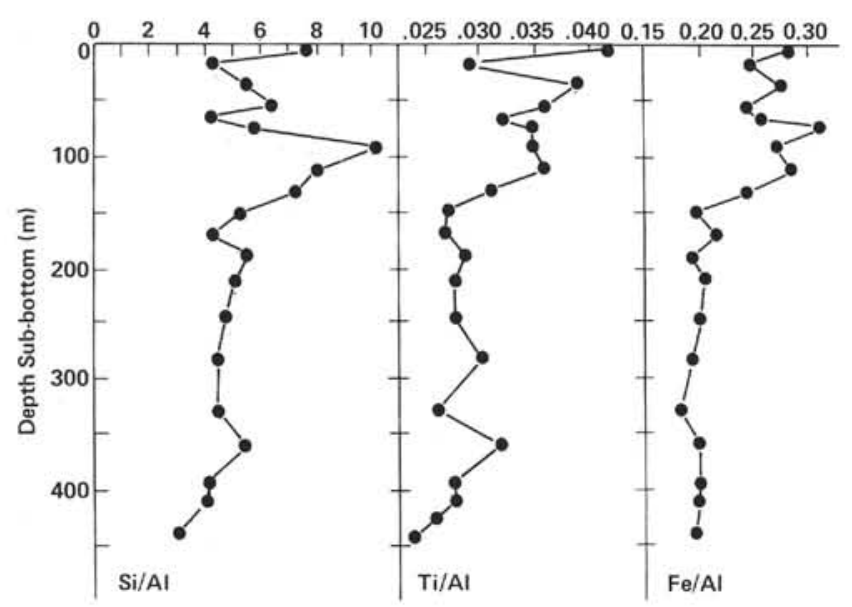

Figure 12. Elemental atomic ratios to aluminum of bulk sediments at Site 479 .

( $\sim 600 \mathrm{~m} / \mathrm{m} . \mathrm{y}$.$) , which implies that below about 15 \mathrm{me}-$ ters sub-bottom the sediment acts as a closed system (Gieskes, 1975). Sulfate reduction is essentially complete below 10 meters, that is, constant sulfate concentrations of $3 \mathrm{mM} \pm 1$ are reached. It is not certain why nonzero sulfate values are obtained, but sea-water contamination $(\approx 10 \%)$ cannot be ruled out completely in these disturbed, gaseous sediments. Generally methane production occurs only when sulfate reduction processes are completed. However, it is possible that these processes will occur when sulfate reduction processes have ceased, even at nonzero sulfate concentrations. Be- low 10 meters depth the production of methane and higher hydrocarbons become increasingly important ( $\mathrm{Si}$ moneit, this volume, Pt. 2). Alkalinity values reach levels higher than $80 \mathrm{meq} / \mathrm{l}$ and dissolved ammonia values increase gradually to reach concentrations as high as $\mathbf{2 6}$ $\mathrm{mM}$ at 250 meters depth. Again, as at all other Leg 64 sites, dissolved phosphate rapidly increases below the sediment surface, with a maximum occurring at about 25 meters depth.

As a result of the production of ammonia by the decomposition of organic carbon, a subsurface maximum in dissolved magnesium again occurs. The rapid decrease in $\mathrm{Mg}^{++}$below 50 meters is probably related to the formation of dolomite in these sediments. The first well-defined layer of dolomite occurs at about 88.5 meters. Dissolved calcium typically shows a minimum as a result of carbonate precipitation reactions, but only in the deeper sections are concentrations slightly above that of sea water observed. Agreement between dissolved calcium data obtained from routine squeezing techniques and by means of the in situ samples is excellent, as is the agreement for dissolved magnesium, indicating the essential validity of the shipboard squeezing procedure.

Dissolved strontium concentrations increase rapidly with depth, which may in part be due to ion exchange processes as well as to carbonate recrystallization processes. The carbonate contents in the sediments, however, are generally very low (Simoneit, this volume, Pt. 2). The data on the strontium isotope composition indicate little or no contribution of volcanic material. 
Data on dissolved lithium indicate a very rapid increase to very high concentrations, with the in situ concentrations being slightly less than the concentrations obtained from routine samples. This phenomenon clearly shows that high lithium concentrations are not necessarily caused by hydrothermal reactions alone. The source of lithium is most likely located in the siliceous phases (opal-A, diatoms), which can contain substantial amounts of lithium (B. Fenical, pers. comm.). Dissolved silica concentrations in interstitial water appear close to saturation with respect to opaline silica, but it is difficult to estimate how much opal dissolution and subsequent removal of silica to other mineral phases has occurred. Further work on the lithium concentrations in the solid phases is planned to assess this problem.

Again dissolved chloride indicates a distinct minimum at 125 meters, more pronounced than at Sites 478 and 481 . We do not think the minima can be related to the occurrence of aquifers in these sediments, particularly not at Site 481 . It is doubtful that the increases in chloride are due to dilution effects resulting from organic carbon decomposition. Organic carbon contents throughout the site vary between 2 and $4 \% \mathrm{C}$, and we estimate that only a relatively small part of this organic carbon is decomposed in the upper 200 meters. Simple mass balance calculations then indicate that the observed dilution effect is difficult to understand in terms of organic matter diagenesis. We are left with the speculation that the minima represent a paleosalinity signal, recording an event of substantially reduced salinities in the Gulf of California. Careful work on the distribution of ${ }^{18} \mathrm{O} /{ }^{16} \mathrm{O}$ and $\mathrm{D} / \mathrm{H}$ isotopes of the interstitial waters may elucidate this problem. The idea that the minimum represents a paleosalinity signal is reinforced by the more pronounced minimum occurring at the shallowest site (Site 479). Unfortunately, at this stage no precise age is available for the minimum at these sites, and such information is necessary to determine whether such a postulated event occurred simultaneously at both.

The data on the bulk sediment composition (Fig. 12) indicate a distinct change in the regime of sedimentation at Site 479, with the sediments between 0 and 150 meters showing much higher ratios of $\mathrm{Ti} / \mathrm{Al}$ and $\mathrm{Fe} / \mathrm{Al}$, similar to the ratios observed in the Guaymas Basin. If the accumulation rates are calculated from diatom data (option $479[\mathrm{~b}]$ in Fig. 2), a break in the sedimentation pattern is indeed observed at $\sim 150$ meters depth. Thus a large change in the sedimentation pattern has occurred at about the same level that the chloride minimum is recorded. At this stage we cannot state whether there is causal relation between these observations.

In Table 8 the atomic elemental ratios of a series of special samples taken from the dolomite layers of Site 479 are presented. In these samples, $\mathrm{Ti} / \mathrm{Al}, \mathrm{K} / \mathrm{Al}$, and $\mathrm{Si} / \mathrm{Al}$ ratios are generally lower than in the remainder of the sediments (cf. Table 5 and Fig. 12). This may indicate slight changes in the mineralogy of associated clay minerals or that in addition to the formation of protodolomites diagenetic changes in the aluminosilicates have also occurred. We have made an attempt to estimate the composition of the dolomites from the ele- mental ratios in Table 8 as follows: First, the "average noncarbonate" $\mathrm{Fe} / \mathrm{Al}, \mathrm{Mg} / \mathrm{Al}$, and $\mathrm{Ca} / \mathrm{Al}$ ratios are determined from the data in Tables 5 and 6 at low $\mathrm{Ca} / \mathrm{Al}$ ratios; second, these ratios are subtracted from the elemental ratios in Table 8, and these corrected ratios are then considered to be the result of the protodolomites. This procedure then results in the computation of the carbonate composition given in Table 8 . Whereas the $\% \mathrm{CaCO}_{3}$ remains relatively constant, it appears that $\mathrm{MgCO}_{3}$ and $\mathrm{FeCO}_{3}$ are interchangeable. No consistent downhole trend in the $\mathrm{MgCO}_{3} / \mathrm{FeCO}_{3}$ ratio is apparent. From the general lack of calcium gradients in the interstitial water column we suggest that calcium carbonate has undergone in situ alteration to protodolomite, with the $\mathrm{Mg}^{++}$and $\mathrm{Fe}^{++}$being generated from the surrounding sediments during diagenesis. As suggested before, mobilization of $\mathrm{Mg}^{++}$from ion exchange positions would have caused the $\mathrm{Mg}^{++}$migration, whereas $\mathrm{Fe}^{++}$would be produced by diagenetic processes involving organic matter decomposition in these reducing sediments. The increase in dissolved strontium (constant ${ }^{87} \mathrm{Sr} /{ }^{86} \mathrm{Sr}$ ) is probably due to release of $\mathrm{Sr}^{++}$from calcite during the formation of protodolomite.

\section{Site 481 (Figs. 13 and 14)}

Site 481 was drilled in the Northern Trough of the Guaymas Basin. Again the sediments are characterized by rapidly accumulated, siliceous, terrigenous sediments with several megaturbidite deposits at 50 meters and 100 meters depth. At 170-195 meters sub-bottom a series of sill intrusions occurs.

The interstitial water data in the upper 150 meters show a clear non-steady-state distribution with minima in alkalinity, ammonia, and phosphate occurring around 45-50 meters. These minima may be related to reduced biogenic activity in the zones of megaturbidites. Values of alkalinity in the upper section are among the highest recorded hitherto in DSDP cores (cf. Cook, 1974; Moore and Gieskes, 1980). Again the production of ammonia has led to the occurrence of one or two maxima in dissolved magnesium, with possible maxima in strontium, potassium, and rubidium.

Below 125 meters the influence of the dolomitedoleritic sill complex becomes most evident. A sharp rise in dissolved strontium occurs just above and below the sill complex. In addition, sharp decreases in the ${ }^{87} \mathrm{Sr} /{ }^{86} \mathrm{Sr}$ ratio are observed. The increases in dissolved chloride and rubidium also testify for the influence of hydrothermal processes on the surrounding sediments. A study of the carbon and oxygen isotopic composition of carbonates above the sill indicate that temperatures as high as $170^{\circ} \mathrm{C}$ may have occurred just above the sill (Kastner, this volume, Pt. 2). During the period that such elevated temperatures occurred the sediment and basalts underwent hydrothermal alteration, as is also evident from the low opaline silica contents (low dissolved silica) and the recrystallization of clay minerals (Kastner, this volume, Pt. 2). The temperatures prevailing must have been elevated enough to cause increases in both dissolved lithium and rubidium. Decreases both in dissolved lithium and potassium toward the sill imply 

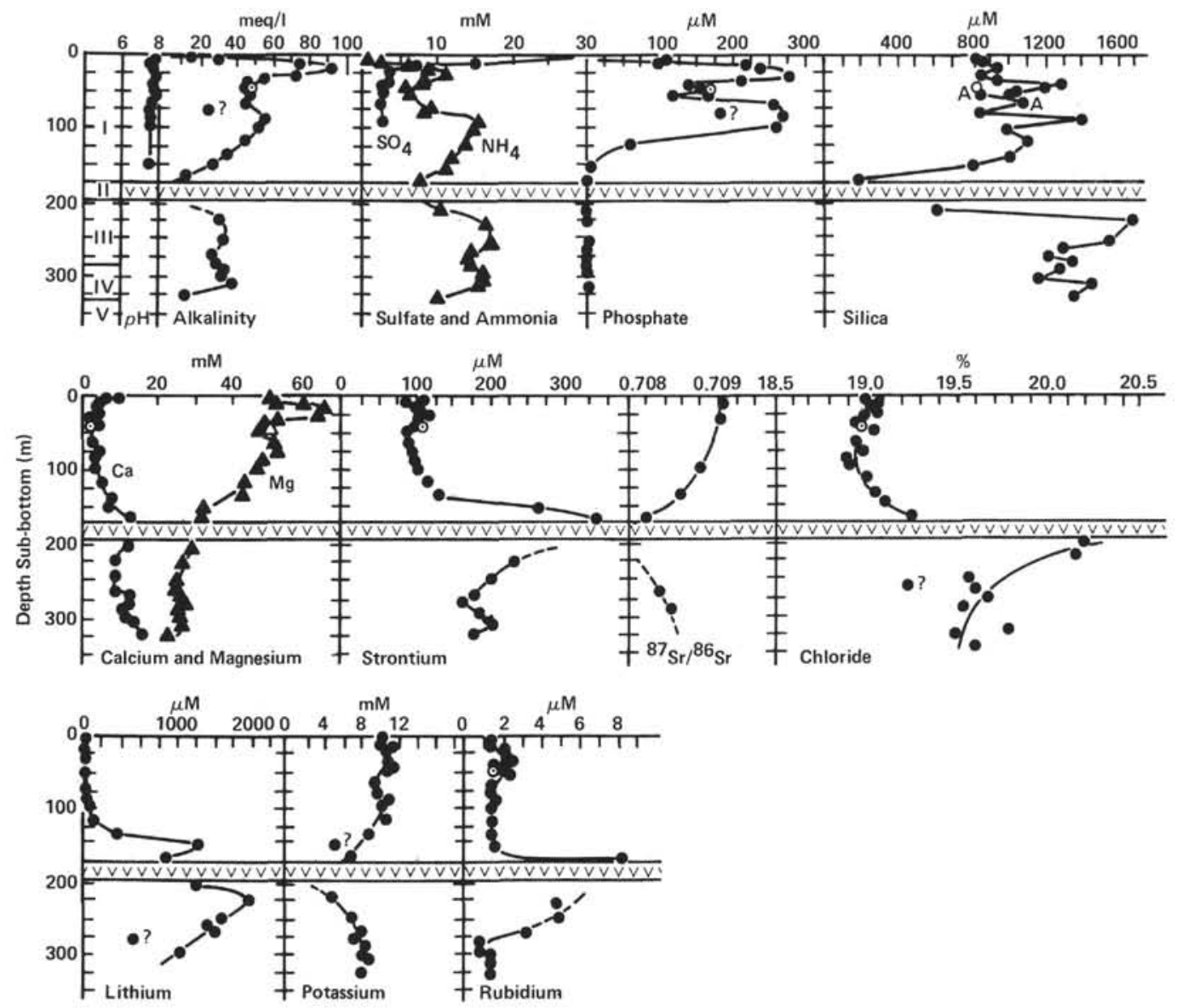

Figure 13. Interstitial water chemistry, Site 481. Lithology: I, alterations between muddy diatomaceous ooze, turbidites, and diatomaceous muds; II, dolerite and basalt sills; III, diatomaceous muds and turbidites; IV, two mass flows, laminated ooze and sill; $\mathrm{V}$, basalt sills in muddy oozes. (Open circles and triangles are in situ data.)

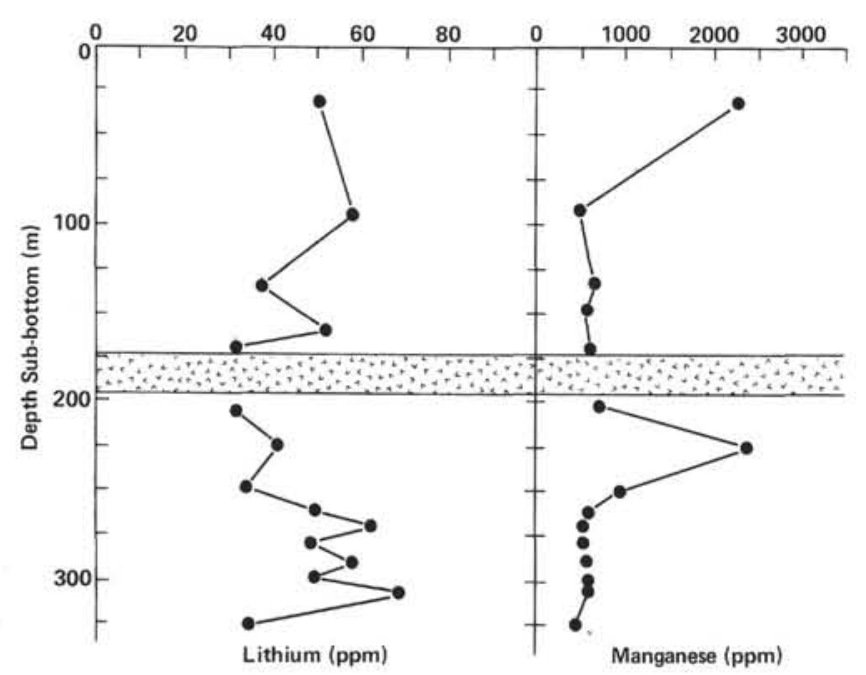

Figure 14. Lithium and manganese in bulk sediments at Site 481.

uptake into the sill during subsequent low-temperature alteration reactions. The distribution of $\delta^{18} \mathrm{O}$ in the interstitial waters shows high values below the sill complex (Fig. 15). These elevated values must be the result of hydrothermal alteration of the sills and/or the sediments surrounding the sills (M. Kastner, pers. comm.). The data do not allow us to speculate on a possible decrease in $\delta^{18} \mathrm{O}$ near the sill; this would be expected from lowtemperature alteration reactions.

\section{GENERAL DISCUSSION}

\section{Early Diagenetic Processes}

In all of the sites drilled in the Gulf of California, sediment accumulation rates and organic carbon contents are higher than found in more open ocean environments. For these reasons the bacterial processes of sulfate reduction and of methane formation, which generally occur after cessation of the sulfate reduction processes, are of great importance. Only at Sites 475 and 476 , which are characterized by much slower accumulation rates, does the sulfate reduction process never cease, and typically no significant production of methane is observed. Chemical changes caused in the interstitial waters by these processes of bacterial degradation of organic matter will be considered as "early diagenetic" in accord with similar changes observed in much shorter cores in the near-shore environment.

Both the sulfate reduction processes and the methane fermentation processes produce large increases in the bicarbonate (alkalinity) concentrations as well as large amounts of ammonium ions. In the upper 50 meters or 
Table 10. Oxygen isotope composition of interstitial waters, Sites 474 and 481 .

\begin{tabular}{|c|c|c|}
\hline Hole/Core/Section & $\begin{array}{l}\text { Depth } \\
\text { (m) }\end{array}$ & ${ }^{18} \mathrm{O}$ (SMOW) \\
\hline $474-1-1$ & 1 & $-0.4 \pm 0.2$ \\
\hline $474-2-1$ & 3 & -0.1 \\
\hline $474-3-2$ & 14 & -0.7 \\
\hline $474-5-2$ & 33 & -0.6 \\
\hline $474-7-3$ & 54 & -0.5 \\
\hline $474-16-2$ & 138 & $-1.1 \pm 0.1$ \\
\hline $474-17-5$ & 152 & $-0.5 \pm 0.1$ \\
\hline $474 \mathrm{~A}-1-2$ & 166 & -1.5 \\
\hline $474 A-4-4$ & 198 & $-0.9 \pm 0.1$ \\
\hline $474 A-7-2$ & 223 & $-1.2 \pm 0.2$ \\
\hline $474 \mathrm{~A}-10-1$ & 250 & $-1.7 \pm 0.1$ \\
\hline $474 \mathrm{~A}-13-3$ & 282 & $-2.0 \pm 0.1$ \\
\hline $474 \mathrm{~A}-16-4$ & 312 & $-1.9 \pm 0.1$ \\
\hline $474 \mathrm{~A}-19-1$ & 328 & $-1.5 \pm 0.2$ \\
\hline $474 \mathrm{~A}-23-3$ & 367 & -1.7 \\
\hline $474 \mathrm{~A}-27-4$ & 407 & $-1.0 \pm 0.1$ \\
\hline $474 \mathrm{~A}-30-2$ & 432 & $-1.3 \pm 0.1$ \\
\hline $474 \mathrm{~A}-33-3$ & 461 & $-1.0 \pm 0.1$ \\
\hline $474 \mathrm{~A}-36-1$ & 488 & $-0.8 \pm 0.1$ \\
\hline $474 \mathrm{~A}-39-1$ & 516 & -1.2 \\
\hline $474 \mathrm{~A}-41-4$ & 540 & -0.9 \\
\hline $481-2-1$ & 5 & $-0.2 \pm 0.1$ \\
\hline $481-3-1$ & 10 & -0.1 \\
\hline $481-4, C \mathrm{C}$ & 16 & -0.3 \\
\hline $481-7-2$ & 30 & 0.0 \\
\hline $481-9-1$ & 39 & -0.4 \\
\hline $481-11-2$ & 50 & -0.3 \\
\hline $481 \mathrm{~A}-1-1$ & 43 & 0.0 \\
\hline 481A-3-1 & 62 & $0.0 \pm 0.1$ \\
\hline $481 \mathrm{~A}-4-2$ & 81 & -0.3 \\
\hline $481 A-5-5$ & 87 & $-0.3 \pm 0.1$ \\
\hline $481 \mathrm{~A}-6-4$ & 95 & -0.2 \\
\hline $481 \mathrm{~A}-8-5$ & 115 & $-0.3 \pm 0.1$ \\
\hline $481 \mathrm{~A}-10-4$ & 133 & $0.2 \pm 0.1$ \\
\hline $481 \mathrm{~A}-12-1$ & 147 & $0.7 \pm 0.1$ \\
\hline $481 \mathrm{~A}-14-3$ & 169 & 0.4 \\
\hline $481 \mathrm{~A}-20-1$ & 223 & 1.9 \\
\hline $481 \mathrm{~A}-22-6$ & 250 & 2.0 \\
\hline $481 \mathrm{~A}-24-5$ & 267 & $2.0 \pm 0.1$ \\
\hline $481 \mathrm{~A}-25-6$ & 279 & $1.3 \pm 0.1$ \\
\hline $481 \mathrm{~A}-27-7$ & 298 & 1.3 \\
\hline $481 \mathrm{~A}-28-5$ & 305 & 1.0 \\
\hline $481 \mathrm{~A}-30-5$ & 324 & 0.5 \\
\hline
\end{tabular}

less of the sediment column, large increases in dissolved phosphate also occur, but these concentrations dissipate rapidly with depth, presumably as a result of precipitation as phosphates. The amounts of phosphates produced are probably small (organic carbon contents are only $5 \%$ or less) and, therefore, these phosphates will be difficult to detect. As a result of the very high values of alkalinity, carbonate precipitation processes in the form of calcite and perhaps of dolomite (Site 479) can explain the lower values of dissolved calcium and magnesium at greater depths. We do not intend here to expand on stoichiometric calculations of organic matter diagenesis, but we do wish to stress the large-scale production of dissolved ammonia in these sediments.

Previous workers (e.g., Rosenfeld, 1979) have shown that ammonia produced in sediments with high organic carbon contents will affect the concentrations of both exchangeable and nonexchangeable ammonia in the solid phase. In very recent sediments the exchangeable ammonia concentrations are especially affected (Rosenfeld, 1979), but in older sediments the nonexchangeable ammonia concentrations may change appreciably (Stevenson and Tilo, 1970). These sediments, especially at Site 479 , may lend themselves particularly well to the study of exchangeable versus nonexchangeable ammonia, but such studies have not yet been carried out.

Walton (1949) gives the following orders for the absorption of monovalent and divalent cations on cation exchangers: $\mathrm{Cs}^{+}>\mathrm{Rb}^{+}>\mathrm{K}^{+}>\mathrm{NH}_{4}{ }^{+}>\mathrm{Na}^{+}>\mathrm{Li}^{+}$ and $\mathrm{Ba}^{++}>\mathrm{Sr}^{++}>\mathrm{Ca}^{++}>\mathrm{Mg}^{++}$. Typically, in seawater-equilibrated clay mixtures $(80 \%$ illite, $20 \%$ montmorillonite), the exchange complements found for major constituents are $0.52 \mathrm{Na}, 0.28 \mathrm{Mg}, 0.11 \mathrm{Ca}$, and 0.10 K (Sayles and Mangelsdorf, 1977). These complements will change with interstitial water composition and certainly when $\mathrm{NH}_{4}{ }^{+}$ions are introduced in large amounts. Unfortunately, no information is available on the exchange complements of $\mathrm{Li}^{+}, \mathrm{Rb}^{+}$, and $\mathrm{Sr}^{++}$. Though these ions are only minor constituents of sea water, exchange reactions may still be important. Thus, introduction of relatively large quantities of dissolved ammonia ( $>10 \mathrm{mM}$ in Leg 64 sites) is likely to result in ion exchange reactions with the solid phases. Until Leg 64 only two sites have ever shown reversals in the concentrations of dissolved constituents in their concentrationdepth profiles: Site 262 (Timor Trough; Cook, 1974) indicated intermediate maxima in $\mathrm{Mg}^{++}$and less equivocally in $\mathrm{K}^{+}$and $\mathrm{Ba}^{++}$, and Site 440 (Japan Trench; Moore and Gieskes, 1980) showed a clear maximum in dissolved magnesium associated with very high $\mathrm{NH}_{4}{ }^{+}$values. Most sites drilled during Leg 64 show intermediate maxima in $\mathrm{K}^{+}, \mathrm{Rb}^{+}, \mathrm{Mg}^{++}$and perhaps $\mathrm{Sr}^{++}$; the effect on dissolved magnesium and potassium is the most discernible. These phenomena are especially clear at Site 474 , where maxima in $\mathrm{K}^{+}, \mathrm{Rb}^{+}$, and $\mathrm{Mg}^{++}$ are well defined (Fig. 3). No apparent ion exchange effect on dissolved lithium has been observed, suggesting a relatively small exchange complement of this ion on the clays.

Why do only relatively few sites show these maxima in cations? At DSDP sites characterized by sedimentation rates of $>200 \mathrm{~m} / \mathrm{m}$.y., the sediment column becomes essentially a closed system at sub-bottom depths of less than 45 meters (Gieskes, 1975). Such sediments are often characterized by high organic carbon contents with associated high rates of chemical decomposition reactions involving organic matter. When such reaction rates are relatively high, diffusion processes cannot smooth out any concentration gradients caused in the interstitial waters by such reactions (Lasaga and Holland, 1976). This, therefore, appears to be the case at the sites at which these concentration reversals in cations have been observed. The maxima occur in the upper part of the methane production zone, where concentrations and rates of production of dissolved ammonia are very high. In the Leg 64 sediments the observed maxima occur below depths of 20 meters. In principle, these concentration reversals should also be observable in near-shore sediments with very high accumulation 

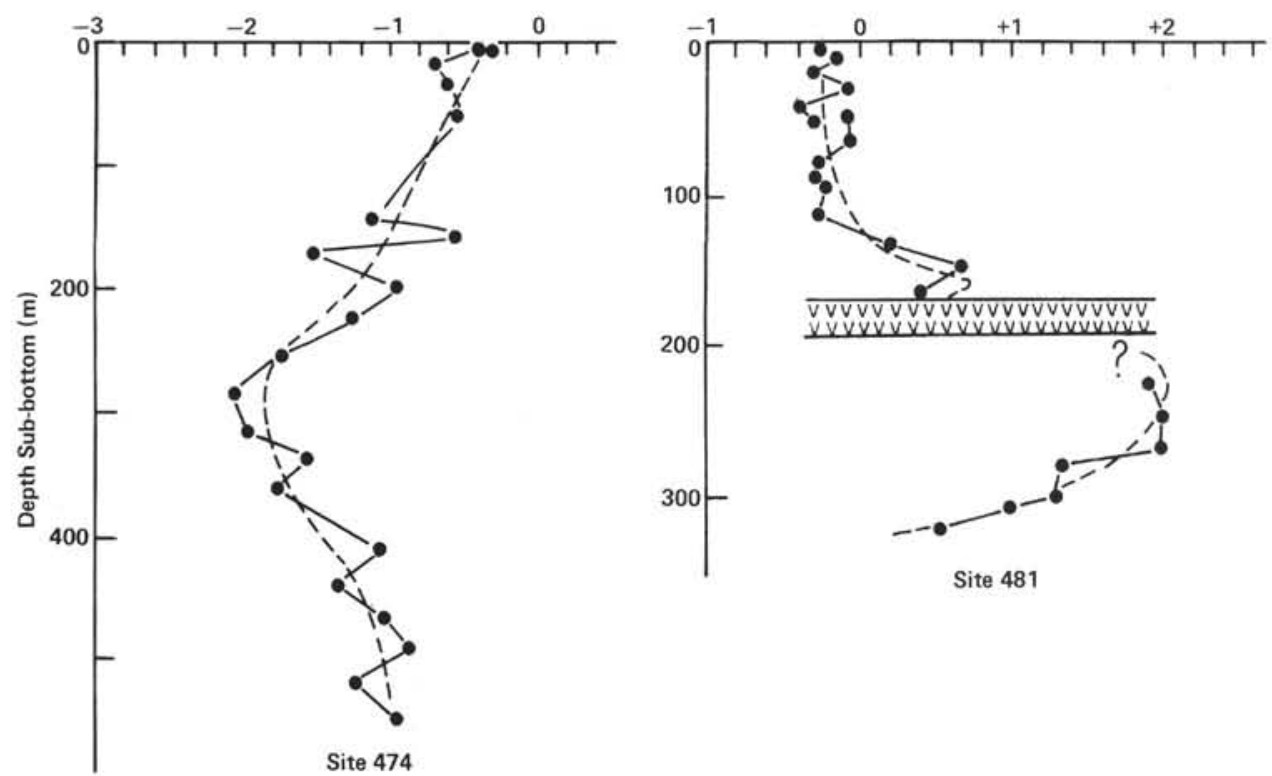

Figure $15 .{ }^{18} \mathrm{O} /{ }^{16} \mathrm{O}$ in interstitial waters at Sites 474 and 481 . Dotted line represents trend line.

rates, within the normal "piston coring range" at depths of less than 20 meters.

Of some interest is the occurrence of a salinity maximum, mostly caused by the high alkalinity $\left(\mathrm{HCO}_{3}^{-}\right)$ values, in the zone of the observed concentration reversals. Below this salinity maximum, salinities decrease rapidly to values typical of interstitial waters depleted in sulfate and alkalinity (Gieskes, 1974).

\section{Hydrothermal Alteration of Sediments and/or Basalts}

The drilling of Sites 477,478 , and 481 in the Guaymas Basin afforded a unique opportunity to study the effects of doleritic sill intrusions on hydrothermal processes in the sediments of the Guaymas Basin (Einsele et al., 1980). Site 477 in the Southern Trough represents a system in which hydrothermal activity is presently occurring in the sediment column below the dolerite sill intrusions between 30 and 100 meters sub-bottom. The heat source must be located below the cored section. Site 478 , located near the transform fault connecting the Southern Trough and the Northern Trough, does show the presence of sills, but these intrusions are presumably old; because of the location of the site no new intrusions are to be expected there. Site 481 is situated in the Northern Trough, but at some distance from the heat flow anomaly described by Williams et al. (1979). At this site the interstitial water data (Fig. 13) clearly indicate that the uppermost sill complex represents the most recent intrusion.

Work on the hydrothermal alteration occurring during high-temperature interaction between basalts and sea water (Bischoff and Dickson, 1975; Bischoff and Seyfried, 1978; Hajash, 1975; Mottl and Holland, 1978; Seyfried and Bischoff, 1979; Edmond et al., 1979) has indicated that, at temperatures above $200^{\circ} \mathrm{C}$, magnesium and sulfate are generally removed quantitatively from sea water, whereas elements such as $\mathrm{Li}^{+}, \mathrm{K}^{+}$, $\mathrm{Ca}^{++}$are released. Evidence from altered basalts (Hum- phris and Thompson, 1978) indicates that some of these elements, for example $\mathrm{Li}^{+}$and particularly $\mathrm{Sr}^{++}$, are precipitated in hydrothermal phases, such as epidote. At lower temperatures some of these trends reverse. For instance, Seyfried and Bischoff (1978) showed that between $75^{\circ}$ and $150^{\circ} \mathrm{C}$ a reversal occurs for dissolved potassium, which at lower temperatures is removed from sea water. J. L. Bischoff and W. Seyfried kindly made available some samples from their hydrothermal experiments, and we carried out analyses for dissolved lithium and rubidium (Table 11). These data suggest that at temperatures between $150^{\circ}$ and $200^{\circ} \mathrm{C}$ a reversal occurs for lithium - that is, at lower temperatures uptake of lithium occurs into the basalts. The data for rubidium suggest release of $\mathrm{Rb}^{+}$to sea water even at temperatures as low as $150^{\circ} \mathrm{C}$. The quenched samples suggest rapid uptake of $\mathrm{Rb}^{+}$upon cooling. Finally, at low temperatures, reaction rates are so slow that no significant change in $\mathrm{Li}^{+}$or $\mathrm{Rb}^{+}$occurs even after 27 months. Water/rock ratios in sediments are generally much lower than in experimental systems, and thus much larger

Table 11. $\mathrm{Li}^{+}$and $\mathrm{Rb}^{+}$concentration, sea-water-basalt experiments. ${ }^{\mathrm{a}}$

\begin{tabular}{clcl}
\hline $\begin{array}{c}\text { Temperature } \\
\left({ }^{\circ} \mathrm{C}\right)\end{array}$ & \multicolumn{1}{c}{ Sample } & $\begin{array}{c}\mathrm{Li} \\
(\mu \mathrm{M})\end{array}$ & $\begin{array}{c}\mathrm{Rb} \\
(\mu \mathrm{M})\end{array}$ \\
\hline $25^{\circ}$ & Sea-water & 27.0 & 1.5 \\
& 25-5-20 mo. & 27.0 & 1.5 \\
& 25-6-27 mo. & 27.0 & 1.5 \\
$150^{\circ}$ & GL-150-10-Quench & 22.8 & 1.3 \\
& GL-150-10-6 1968 hr. & 21.6 & 2.3 \\
$200^{\circ} \mathrm{C}$ & GL-200-10 Quench & 44.3 & 1.4 \\
& GL-200-10-3 192 hr. & 46.8 & 2.85 \\
$300^{\circ} \mathrm{C}$ & GL-300-10 Quench & 173 & 1.3 \\
& GL-300-10-3 240 hr. & 94.2 & 2.2 \\
& GL-300-10-5 1296 hr. & 92.7 & 3.0 \\
\hline
\end{tabular}

a Samples provided by J. L. Bischoff and W. Seyfried. 
changes occur in the concentration of Sites 477-481 and in oceanic hot springs (Edmond et al., 1979; Menzies and Seyfried, 1979).

The interstitial water chemistry in the Guaymas Basin sites is in qualitative agreement with these observations. At Site 477 , where temperatures in excess of $200^{\circ} \mathrm{C}$ occur at the bottom of the site, enrichments are typically observed in $\mathrm{Li}^{+}, \mathrm{K}^{+}, \mathrm{Rb}^{+}, \mathrm{Ca}^{++}$, and $\mathrm{Sr}^{++}$. As discussed later, these concentration changes are different from those observed in the Galapagos hot springs (Edmond et al., 1979), not only because of different waterrock ratios but also because concentrations of $\mathrm{Li}, \mathrm{K}$, and $\mathrm{Rb}$ are much higher in sediments than in basalts. At Site 481 , where the sills presently have temperatures between 30 and $35^{\circ} \mathrm{C}$, uptake of $\mathrm{K}^{+}$and $\mathrm{Li}^{+}$occurs in the dolerite, as is indicated by the interstitial water profiles. However, evidence from recrystallized calcites (M. Kastner, pers. comm.) indicates that temperatures higher than $167^{\circ} \mathrm{C}$ must have prevailed in the sediments during the period that the sill intrusions occurred. These processes would have caused increases in $\mathrm{Li}^{+}, \mathrm{Rb}^{+}$, and $\mathrm{K}^{+}$in the interstitial waters surrounding the sills. After the sills cooled, however, reversals in these concentrations would occur as a result of "retrograde" reactions. Thus the anomalies caused by the high-temperature interactions between basalts-sediment-pore waters are in a state of decay at this site. At Site 478 the only profile that still shows the hydrothermal signal is the $\mathrm{Li}^{+}$concentration profile. In addition to these alkali cations, the hydrothermal interaction is also noticeable in the dissolved chloride and the ${ }^{18} \mathrm{O} /{ }^{16} \mathrm{O}$ profiles (Kastner, this volume, $\mathrm{Pt}$. 2).

Chemical evidence on the solid phases of Site 477 indicates that the sediments are to a large extent the sources of dissolved $\mathrm{Li}^{+}, \mathrm{K}^{+}$, and $\mathrm{Rb}^{+}$. Data on $\delta^{18} \mathrm{O}$ of the bulk silicates (Kastner, this volume) support this contention. Data on the ${ }^{87} \mathrm{Sr} /{ }^{86} \mathrm{Sr}$ composition of dissolved strontium and of the solid phases indicate a possible basaltic contribution to the dissolved strontium. The ${ }^{87} \mathrm{Sr} /{ }^{86} \mathrm{Sr}$ data of the strontium dissolved in the interstitial water are summarized in Figure 16, in which the data for Site 479 serve as reference values.

That the interstitial waters also record a signal caused by hydrothermal interactions with basalts is confirmed by the finding of an excess ${ }^{3} \mathrm{He} /{ }^{4} \mathrm{He}$ ratio in a gas sample collected from Site 477 , at 144 meters sub-bottom depth.

\section{ACKNOWLEDGMENTS}

We wish to thank the scientific staff and technicians on board Glomar Challenger for their cooperation in this geochemical program. We thank Mr. Jim Pine, the shipboard chemist, whose tolerance of J.M.G.'s presence in the ship's laboratory is appreciated.

This research was generously supported by NSF Grant OCE 7724102 (J.M.G) as well as by the National Environmental Research Council of Great Britain (H.E.).

Dr. R. E. McDuff critically reviewed the manuscript.

\section{REFERENCES}

Albarede, F., Vitrac-Michard, A., Minster, J. F., and Michard, G., 1980. Strontium isotope composition in hydrothermal systems. Trans. Am. Geophys. Union (EOS), 61:994-995.

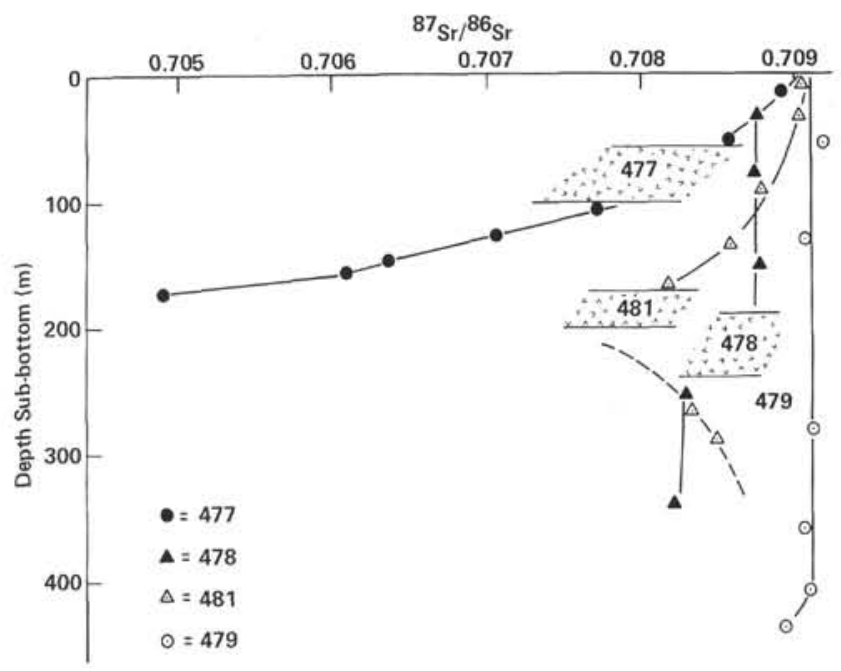

Figure $16 .{ }^{87} \mathrm{Sr} /{ }^{86} \mathrm{Sr}$ of dissolved strontium at Sites $477,478,479,481$.

Barnes, R. O., Gieskes, J. M., Horvath, J., and Akiyama, W., 1979. Interstitial water studies, Legs 47A, B. In Sibuet, J.-C., Ryan, W. B. F., et al., Init. Repts. DSDP, 47, Pt. 2: Washington (U.S. Govt. Printing Office), 577-582.

Berner, R. A., 1980. Early Diagenesis: A Theoretical Approach. Princeton Series in Geochemistry.

Bischoff, J. L., and Dickson, F. W., 1975. Seawater-basalt interaction at $200^{\circ} \mathrm{C}$ and 500 bars: Implications for origin of seafloor heavy metal deposits and regulation of seawater chemistry. Earth Plan. Sci. Lett., 25:385-397.

Bischoff, J. L., and Seyfried, W. E., 1978. Hydrothermal chemistry of seawater from $25^{\circ} \mathrm{C}$ to $350^{\circ} \mathrm{C}$. Am. J. Sci., 278:838-860.

Cook, P., 1974. Geochemistry and diagenesis of interstitial fluids and associated calcareous oozes, Deep Sea Drilling Project, Leg 27, Site 262, Timor Trough. In Veevers, J. J., Heirtzler, J. R., et al., Init. Repts. DSDP, 27: Washington (U.S. Govt. Printing Office), 463-480.

Donnelly, T. W., and Wallace, J., 1976. Major- and minor-element chemistry of Antarctic clay-rich sediments. In Hollister, C. D., Craddock, C., et al., Init. Repts. DSDP, 35: Washington (U.S. Govt. Printing Office), 427-446.

Edmond, J. M., Measures, C., McDuff, R. E., Chan, L. H., Collier, R., and Grant, B., 1979. Ridge crest hydrothermal activity and the balances of the major and minor elements in the ocean: The Galapagos data. Earth Plan. Sci. Lett., 46:1-18.

Einsele, G., Gieskes, J. M., et al., 1980. Intrusion of basaltic sills into highly porous sediments, and resulting hydrothermal activity. Nature, 283:441-445.

Epstein, S., and Mayeda, T., 1953. Variations of $\mathrm{O}^{18}$ content of water from natural sources. Geochim. Cosmochim. Acta, 4:213-224.

Flanagan, F. J., 1972. 1972 values of international geochemical references samples. Geochim. Cosmochim. Acta, 37:1189-1200.

Gieskes, J. M., 1973. Interstitial water studies, Leg 15-alkalinity, $p \mathrm{H}, \mathrm{Mg}, \mathrm{Ca}, \mathrm{Si}, \mathrm{PO}_{4}$, and $\mathrm{NH}_{4}$. In Heezen, B. C., MacGregor, I. D., et al., Init. Repts. DSDP, 20: Washington (U.S. Govt. Printing Office), 813-829.

, 1974. Interstitial water studies, Leg 25. In Simpson, E. S. W., Schlich, R., et al., Init. Repts. DSDP, 25: Washington (U.S. Govt. Printing Office), 361-394.

1975. Chemistry of interstitial waters of marine sediments. Ann. Rev. Earth Planet. Sci., 3:433-453.

1981a. Deep sea drilling interstitial water studies: Implications for chemical alteration of the oceanic crust, Layers I and II. In Warme, J. B., Douglas, R. G., Winterer, E. L. (Eds.), The Deep Sea Drilling Project: A Decade of Progress. Society of Economic Paleontologists and Mineralogists Special Publication 32. , 1981b. Interstitial water studies, Deep Sea Drilling Project Leg 60. In Hussong, D. M., Uyeda, S., et al., Init. Repts. $D S D P, 60$ : Washington (U.S. Govt. Printing Office), 749-754. 
Gieskes, J. M., and Johnson, J., 1981. Interstitial water studies, Deep Sea Drilling Project Leg 59. In Kroenke, L., Scott, R., et al., Init. Repts. DSDP, 59: Washington (U.S. Govt. Printing Office), 627-630.

Gieskes, J. M., and Lawrence, J. R., 1976. Interstitial water studies, Leg 35. In Hollister, C. D., Craddock, C., et al., Init. Repts. DSDP, 35: Washington (U.S. Govt. Printing Office), 407-423. in press. Alteration of volcanic matter in deep sea sediments: Evidence from the chemical composition of interstitial waters from deep sea drilling cores. Geochim. Cosmochim. Acta.

Gieskes, J. M., Lawrence, J. R., and Galleisky, G., 1979. Interstitial water studies, Leg 38. In Talwani, M., Udintsev, G., et al., Init. Repts. DSDP, Suppl. to Vols. 38, 39, 40, and 41: Washington (U.S. Govt. Printing Office), 121-133.

Hajash, A., 1975. Hydrothermal processes along mid-ocean ridges: An experimental investigation. Contrib. Mineral. Petrol., 53: 205-226.

Hawkesworth, C. J., and Elderfield, H., 1978. The strontium isotope composition of interstitial waters from Sites 245 and 336 of DSDP. Earth Plan. Sci. Lett., 40:423-432.

Humphris, S. E., and Thompson, G., 1978. Trace element mobility during hydrothermal alteration of oceanic basalts. Geochim. Cosmochim. Acta, 42:127-136.

Kastner, M., and Gieskes, J. M., 1976. Interstitial water profiles and sites of diagenetic reactions, Leg 35, DSDP, Bellingshausen Abyssal Plain. Earth Plan. Sci. Lett., 33:11-20.

Kastner, M., Keene, J. B., and Gieskes, J. M., 1977. Diagenesis of siliceous oozes. I. Chemical controls on the rate of opal-A to opalCT transformation-an experimental study. Geochim. Cosmochim. Acta, 41:1041-1059.

Lasaga, A. C., and Holland, H. D., 1976. Mathematical aspects of non-steady state diagenesis. Geochim. Cosmochim. Acta, 40: 257-266.

Lawver, L. A., Williams, D. L., and Von Herzen, R. P., 1975. A major geothermal anomaly in the Gulf of California. Nature, 257: 23-28.

Lupton, J. E., 1979. Helium-3 in the Guaymas Basin: Evidence for injection of mantle volatiles in the Gulf of California. J. Geophys. Res., 84:7446-7452.

Manheim, F. T., and Sayles, F. L., 1974. Composition and origin of interstitial waters of marine sediments based on deep sea drill cores. In Goldberg, E. D. (Ed.), The Sea (Vol. 5): New York (Wiley-Interscience), 527-568.

Mann, R., and Gieskes, J. M., 1975. Interstitial water studies, Leg 28. In Hayes, D. E., Frakes, L. A., et al., Init. Repts, DSDP, 28 : Washington (U.S. Govt. Printing Office), 805-814.

Menzies, M., and Seyfried, W. E., 1979. Basalt-seawater interaction: Trace element and strontium isotopic variations in experimentally altered glassy basalt. Earth Plan. Sci. Lett., 44:463-472.

Moore, D. G., 1973. Plate-edge deformation and crustal growth, Gulf of California structural province. Geol. Soc. Am. Bull., 84: 1883-1906.

Moore, G. W., and Gieskes, J. M., 1980. Interaction between sediment and interstitial waters near the Japan Trench, Deep Sea Drilling Project Leg 57, In Scientific Party, Init. Repts. DSDP, 56, 57, Pt. 2: Washington (U.S. Govt. Printing Office), 1269-1275.

Mottl, M. J., and Holland, H. D., 1978. Chemical exchange during hydrothermal alteration of basalt by seawater. I. Experimental results for major and minor components of seawater. Geochim. Cosmochim. Acta, 42:1103-1115.

Rosenfeld, J. K., 1979. Ammonium adsorption in nearshore anoxic sediments. Limnol. Oceanogr., 24:356-364.

Sayles, F. L., and Mangelsdorf, P. G., 1977. The equilibration of clay minerals with seawater: Exchange reactions. Geochim. Cosmochim. Acta, 41:951-960.

Sayles, F. L., and Manheim, F. T., 1975. Interstitial solutions and diagenesis in deeply buried marine sediments: Results from the Deep Sea Drilling Project. Geochim. Cosmochim. Acta., 39: 103-127.

Sayles, F. L., Manheim, F. T., and Waterman, L. S., 1973. Interstitial water studies on small core samples, Leg 15. In Heezen, B. C., MacGregor, I. D., et al., Init. Repts. DSDP, 20: Washington (U.S. Govt. Printing Office), 783-804.
Seyfried, W. E., and Bischoff, J. L., 1979. Low temperature basalt alteration by seawater: An experimental study at $70^{\circ} \mathrm{C}$ and $150^{\circ} \mathrm{C}$. Geochim. Cosmochim. Acta, 43:1937-1947.

Shapiro, L., and Brannock, W. W., 1962. Rapid Analysis of Silicate, Carbonate and Phosphate Rocks. U.S. Geol. Survey Bull. 1144A.

Stevenson, F. J., and Tilo, S. N., 1970. Nitrogenous constituents of deep sea sediments, In Hobson, G. C., and Speers, G. C. (Eds.), Advances in Organic Geochemistry: Pergamon, pp. 237-263.

van Andel, Tj. H., and Shor, G. G., 1964. Marine Geology of the Gulf of California. Am. Assoc. Pet. Geol. Mem. 3.

Walton, H. F., 1949. Ion exchange equilibria. In Nachod, F. C. (Ed.), Ion Exchange, Theory and Application: New York (Academic Press), pp. 3-28.

Williams, D. L., Becker, K., Lawver, L. A., and Von Herzen, R. P., 1979. Heat flow at the spreading centers of the Guaymas Basin, Gulf of California. J. Geophys. Res., 84:6757-6769.

\section{APPENDIX \\ Rubidium Determinations in Interstitial Waters Jeff Johnson}

Rubidium is determined by flameless atomic absorption techniques. In accordance with the method of Katz (1975), sulfuric acid background is used to reduce interferences.

\section{Sample Preparation}

$0.1 \mathrm{~cm}^{3}$ sea-water sample

$0.1 \mathrm{~cm}^{3} 500 \mathrm{mM} \mathrm{H}_{2} \mathrm{SO}_{4}$

$0.1 \mathrm{~cm}^{3} 600 \mathrm{mM} \mathrm{NH}_{4} \mathrm{NO}_{3}$

$0.2 \mathrm{~cm}^{3} 5 \%$ ascorbic acid

$0.5 \mathrm{~cm}^{3}$ double-distilled water

\section{Method}

Solution is swirled thoroughly and $25 \mu \mathrm{m}$ of sample is introduced into the carbon furnace. A Perkin-Elmer model 403 atomic absorption spectrophotometer and model HGA-2200 controller with P-E graphite furnace are used.

Machine setting: Dry $35 \mathrm{~s}$ at $105^{\circ} \mathrm{C}$ Char $25 \mathrm{~s}$ at $900^{\circ} \mathrm{C}$

Atomize $7 \mathrm{~s}$ at $2700^{\circ} \mathrm{C}$

Flow rate $=$ Stop 3

Wavelength $=390$

The Rb-electrodeless discharge lamp is used. Data are recorded on a chart recorder.

Standards

Spiked La Jolla pier water and IAPSO standard sea water are used.

Notes

1. Good agreement was obtained with mass spectrometric data (H. Elderfield, pers. comm.).

2. $\mathrm{H}_{2} \mathrm{SO}_{4}$ : The signal strength is strongly dependent on the sulfate concentration below $400 \mathrm{mM}$ of $\mathrm{SO}_{4}=$. Since $\mathrm{SO}_{4}=$ varies in porewater samples we made $\mathrm{SO}_{4}=500 \mathrm{~m} M$

3. $\mathrm{NH}_{4} \mathrm{NO}_{3}$ : This mixes in sample to form $\mathrm{NH}_{4} \mathrm{Cl}$ and $\mathrm{NaNO}_{3}$. In the latter form $\mathrm{Na}$ and $\mathrm{Cl}$ will volatilize at lower temperatures and thus they are effectively removed from the samples. The signal strength is dependent on the $\mathrm{Na}^{+}$concentration, so that $\mathrm{Na}^{+}$removal is essential.

4. Ascorbic acid: It has been recommended that ascorbic acid be added to improve reproducibility and to enhance signal strength. The ascorbic acid use does improve the signal but does have one drawback. After several burns the carbon of the ascorbic acid leaves a large residue in the carbon furnace, which affects the absorbance zero. The residue is easily removed with a pipe cleaner or Kimwipe (after 2-3 burns).

5. It is very important to pipette reproducibly.

\section{Reference:}

Katz, A., 1975. The determination of rubidium and strontium in silicates by flameless atomic-absorption spectroscopy. Chem. Geol., $16: 15-25$. 